JOURNAL OF THE AMERICAN MATHEMATICAL SOCIETY

Volume 19, Number 1, Pages 1-36

S 0894-0347(05)00505-9

Article electronically published on September 16, 2005

\title{
THE DE RHAM-WITT COMPLEX AND $p$-ADIC VANISHING CYCLES
}

\author{
THOMAS GEISSER AND LARS HESSELHOLT
}

\section{INTRODUCTION}

Let $V$ be a henselian discrete valuation ring with quotient field $K$ of characteristic 0 and perfect residue field $k$ of odd characteristic $p$. Let $X$ be a smooth $V$-scheme of relative dimension $r$, and let $i$ and $j$ denote the inclusion of the special and generic fibers, respectively, as in the cartesian diagram

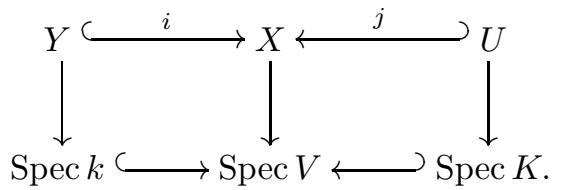

The henselian local ring of $X$ at a generic point of $Y$ is a henselian discrete valuation ring $\mathcal{V}$ whose residue field $\kappa$ is the non-perfect function field of a connected component of $Y$. Let $\mathcal{K}$ be the quotient field of $\mathcal{V}$.

We consider the ring $\mathcal{O}_{X}$ with the log-structure $\alpha: M_{X} \rightarrow \mathcal{O}_{X}$ determined by the special fiber. The absolute de Rham-Witt complex of $\left(X, M_{X}\right)$ [11, 10] is defined to be the universal Witt complex over $\left(\mathcal{O}_{X}, M_{X}\right)$ and is denoted

$$
W_{n} \Omega_{\left(X, M_{X}\right)}^{*}=W_{n} \Omega_{\left(\mathcal{O}_{X}, M_{X}\right)}^{*}
$$

It has a natural descending filtration by the differential graded ideals

$$
\mathrm{Fil}_{U}^{m} W_{n} \Omega_{\left(X, M_{X}\right)}^{*} \subset W_{n} \Omega_{\left(X, M_{X}\right)}^{*}
$$

generated by $W_{n}\left(\mathfrak{m}^{j} \mathcal{O}_{X}\right)$, if $m=2 j$ is even, and by $W_{n}\left(\mathfrak{m}^{j} \mathcal{O}_{X}\right) \cdot d \log _{n} M_{X}$ and by $W_{n}\left(\mathfrak{m}^{j+1} \mathcal{O}_{X}\right)$, if $m=2 j+1$ is odd. Here and throughout $\mathfrak{m}$ denotes the maximal ideal of $V$. There is a natural isomorphism

$$
i^{*} W_{n} \Omega_{\left(X, M_{X}\right)}^{*} / i^{*} \operatorname{Fil}_{U}^{1} W_{n} \Omega_{\left(X, M_{X}\right)}^{*} \stackrel{\sim}{\longrightarrow} W_{n} \Omega_{Y}^{*}
$$

onto the classical de Rham-Witt complex of Bloch-Deligne-Illusie [13] of the special fiber, and there is a natural isomorphism

$$
i^{*} W_{n} \Omega_{\left(X, M_{X}\right)}^{*} / i^{*} \mathrm{Fil}_{U}^{2} W_{n} \Omega_{\left(X, M_{X}\right)}^{*} \stackrel{\sim}{\longrightarrow} W_{n} \Omega_{\left(Y, M_{Y}\right)}^{*}
$$

Received by the editors January 5, 2004.

2000 Mathematics Subject Classification. Primary 11G25, 11S70; Secondary 14F30, 19D55.

Key words and phrases. de Rham-Witt complex, $p$-adic cohomology, algebraic $K$-theory.

A previous version of this paper was entitled On the $K$-theory of a henselian discrete valuation field with non-perfect residue field.

The authors were supported in part by grants from the National Science Foundation. The first author received additional support from the Japan Society for the Promotion of Science and the Alfred P. Sloan Foundation. 
onto the de Rham-Witt complex of Hyodo-Kato 12 of the special fiber with the induced $\log$-structure. (The latter was denoted by $W_{n} \tilde{\omega}_{Y}^{q}$ in op.cit.) But the full complex $W_{n} \Omega_{\left(X, M_{X}\right)}^{*}$ and its quotients by the higher terms of the filtration have not been considered before. In Sect. 1 below we evaluate the graded pieces for the induced filtration of the reduced sheaves

$$
E_{n}^{q}=i^{*} W_{n} \Omega_{\left(X, M_{X}\right)} / p i^{*} W_{n} \Omega_{\left(X, M_{X}\right)}^{q} .
$$

These are quasi-coherent $E_{n}^{0}$-modules on the small étale site of $Y$ which we analyze as follows. A choice of local coordinates of an open neighborhood of $X$ around a point of $Y$ determines a ring homomorphism $\delta_{n}: \mathcal{O}_{Y} \rightarrow E_{n}^{0}$ defined in the corresponding open neighborhood of $Y$. Hence, in this open neighborhood of $Y$, we may consider the sheaves $E_{n}^{q}$ and the graded pieces $\operatorname{gr}_{U}^{m} E_{n}^{q}$ as quasi-coherent $\mathcal{O}_{Y^{-}}$ modules. We show in Thm. 1.3.5 below that both are free $\mathcal{O}_{Y}$-modules and give an explicit basis. We note here that the rank of the free $\mathcal{O}_{Y}$-module $E_{n}^{q}$ is

$$
\operatorname{rk}_{\mathcal{O}_{Y}} E_{n}^{q}=\left(\begin{array}{c}
r+1 \\
q
\end{array}\right) \cdot e \cdot \sum_{s=0}^{n-1} p^{r s}
$$

and that the length of the filtration is $2 e^{\prime}=2 p e /(p-1)$. In Sect. 2 we use this result to analyze the subsheaf $M_{n}^{q}$ of $E_{n}^{q}$ that is fixed by the Frobenius map. The sheaf $M_{n}^{q}$ is defined to be the kernel of the map

$$
R-F: E_{n}^{q} \rightarrow E_{n-1}^{q}
$$

and is a sheaf of abelian groups on the small étale site of $Y$ in the étale topology. The filtration of $E_{n}^{q}$ induces a filtration of $M_{n}^{q}$ and in Thm. 2.1.2 below we evaluate the graded pieces $\operatorname{gr}_{U}^{m} M_{n}^{q}$ considered as sheaves of pro-abelian groups as $n$ varies. The result agrees with the calculation by Bloch-Kato [3, Cor. 1.4.1] of the graded pieces $\operatorname{gr}_{U}^{m} i^{*} R^{q} j_{*} \mu_{p}^{\otimes q}$ of a corresponding filtration of the sheaf of $p$-adic vanishing cycles. By combining the two results we obtain the following theorem.

Theorem A. Suppose $\mu_{p^{v}} \subset K$. Then there is a natural exact sequence

$$
0 \rightarrow i^{*} R^{q} j_{*} \mu_{p^{v}}^{\otimes q} \rightarrow i^{*} W . \Omega_{\left(X, M_{X}\right)}^{q} / p^{v} \stackrel{1-F}{\longrightarrow} i^{*} W . \Omega_{\left(X, M_{X}\right)}^{q} / p^{v} \rightarrow 0
$$

of sheaves of pro-abelian groups on the small étale site of $Y$ in the étale topology.

We expect that Thm. A is valid also if $K$ does not contain the $p^{v}$ th roots of unity. More precisely, we expect that the terms in the sequence satisfy Galois descent for the extension $K\left(\mu_{p^{v}}\right) / K$; compare [14, Thm. 1(1)]. At present, however, the structure of the sheaves $i^{*} W_{n} \Omega_{\left(X, M_{X}\right)}^{q} / p^{v}$ is known only if $K$ contains the $p^{v}$ th roots of unity. It appears to be an important problem to determine the structure of these sheaves in general.

We remark that Thm. A is not valid if the absolute de Rham-Witt complex is replaced by the relative de Rham-Witt complex of Langer-Zink [18. The reader is referred to $[8$ for a comparison of the two complexes.

The algebraic $K$-theory with $\mathbb{Z} / p^{v}$-coefficients of the field $K$ was determined in [10]. In the final Sect. 3 we combine Thm. $\mathrm{A}$ and the main results of [11, 10, to extend this result to the field $\mathcal{K}$. Indeed we prove the following formula predicted by the Beilinson-Lichtenbaum conjectures [1, 2, 19]. 
Theorem B. Suppose that $\mu_{p^{v}} \subset K$. Then the canonical map

$$
K_{*}^{M}(\mathcal{K}) \otimes S_{\mathbb{Z} / p^{v}}\left(\mu_{p^{v}}\right) \rightarrow K_{*}\left(\mathcal{K}, \mathbb{Z} / p^{v}\right)
$$

is an isomorphism.

The second tensor factor on the left-hand side is the symmetric algebra on the $\mathbb{Z} / p^{v}$-module $\mu_{p^{v}}$, which is free of rank one, and the map of the statement takes a generator $\zeta \in \mu_{p^{v}}$ to the associated Bott element $b_{\zeta} \in K_{2}\left(\mathcal{K}, \mathbb{Z} / p^{v}\right)$. The Milnor groups $K_{q}^{M}(\mathcal{K}) / p K_{q}^{M}(\mathcal{K})$ were evaluated by Kato in [15, Thm. 2(1)]. They are concentrated in degrees $0 \leqslant q \leqslant r+2$. Hence Thm. B shows in particular that the algebraic $K$-groups $K_{*}\left(\mathcal{K}, \mathbb{Z} / p^{v}\right)$ are two-periodic above this range of degrees.

The results of this paper were reported in expository form in [9].

In this paper, a pro-object of a category $\mathcal{C}$ will be taken to mean a functor from the set of positive integers, viewed as a category with one arrow from $n+1$ to $n$, to $\mathcal{C}$, and a strict map between pro-objects a natural transformation. A general map between pro-objects $X$ and $Y$ of $\mathcal{C}$ is defined to be an element of

$$
\operatorname{Hom}_{\text {pro }-\mathcal{C}}(X, Y)=\lim _{n} \operatorname{colim}_{m} \operatorname{Hom}_{\mathcal{C}}\left(X_{m}, Y_{n}\right) .
$$

We view objects of $\mathcal{C}$ as constant pro-objects of $\mathcal{C}$. Throughout, the prime $p$ is a fixed odd prime. We abbreviate $e^{\prime \prime}=e /(p-1)$ and $e^{\prime}=p e /(p-1)$.

This paper was written, in part, while the authors visited the University of Tokyo. The second author also visited the Isaac Newton Institute of Mathematical Sciences and Stanford University. We would like to express our sincere gratitude for the financial support and the hospitality that we received. We are particularly grateful to Viorel Costeanu for allowing us to include his proof of the Steinberg relation in the de Rham-Witt complex as an appendix to this paper.

\section{The absolute de Rham-Witt complex}

1.1. We consider the de Rham-Witt complex of $\log -\mathbb{Z}_{(p)}$-algebras introduced in [10, Sect. 3]; see also [11. It generalizes the de Rham-Witt complex of $\log -\mathbb{F}_{p}$-algebras of Hyodo-Kato 12 .

A $\log$-ring $\left(A, M_{A}\right)$ in the sense of [16] is a ring $A$ (in a topos) with a pre-logstructure defined to be a map of monoids $\alpha: M_{A} \rightarrow(A, \cdot)$ from a monoid $M_{A}$ to the underlying multiplicative monoid of $A$. A log-differential graded ring $\left(D, M_{D}\right)$ is a differential graded ring $D$, a pre-log-structure $\alpha: M_{D} \rightarrow\left(D^{0}, \cdot\right)$, and a map of monoids $d \log : M_{D} \rightarrow\left(D^{1},+\right)$ that satisfies that $d \circ d \log =0$ and that for all $x \in M_{D}, d \alpha(x)=\alpha(x) d \log x$. Maps of log-rings and log-differential graded rings are defined in the obvious way.

Let $W_{n}(A)$ be the ring of Witt vectors of length $n$ in $A$. If $\alpha: M_{A} \rightarrow A$ is a pre-log-structure, then so is the composite

$$
M_{A} \stackrel{\alpha}{\rightarrow} A \rightarrow W_{n}(A),
$$

where the right-hand map is the multiplicative section $a \mapsto[a]_{n}=(a, 0, \ldots, 0)$. We denote this log-ring by $\left(W_{n}(A), M_{A}\right)$. By a Witt complex over $\left(A, M_{A}\right)$ we mean the following structure:

(i) a pro-log-differential graded ring $\left(E_{*}^{*}, M_{E}\right)$ and a strict map of pro-log-rings

$$
\lambda:\left(W \cdot(A), M_{A}\right) \rightarrow\left(E_{.}^{0}, M_{E}\right) ;
$$


(ii) a strict map of pro-log-graded rings

$$
F:\left(E_{.}^{*}, M_{E}\right) \rightarrow\left(E_{\cdot-1}^{*}, M_{E}\right)
$$

such that $F \lambda=\lambda F$ and such that

$$
\begin{aligned}
F d \log _{n} \lambda(a) & =d \log _{n-1} \lambda(a), & & \text { for all } a \in M_{A}, \\
F d \lambda\left([a]_{n}\right) & =\lambda\left([a]_{n-1}\right)^{p-1} d \lambda\left([a]_{n-1}\right), & & \text { for all } a \in A ;
\end{aligned}
$$

(iii) a strict map of pro-graded modules over the pro-graded ring $E^{*}$.

$$
V: F_{*} E_{\cdot-1}^{*} \rightarrow E^{*}
$$

such that $V \lambda=\lambda V, F V=p$, and $F d V=d$.

A map of Witt complexes over $(A, M)$ is a strict map of pro-log differential graded rings which commutes with the maps $\lambda, F$ and $V$. We write $R$ for the structure map in the pro-system $E^{*}$. and call it the restriction map. The definining relations imply that $d F=p F d$ and $V d=p d V$, but in general there is no formula for $V F$; see [11, Lemma 1.2.1]. The de Rham-Witt complex

$$
W \cdot \Omega_{\left(A, M_{A}\right)}^{*}
$$

is defined to be the initial Witt complex over $\left(A, M_{A}\right)$. The proof that it exists is given in [11, Thm. A]. The proof also shows that the canonical map

$$
\lambda: \Omega_{\left(W_{n}(A), M_{A}\right)}^{q} \rightarrow W_{n} \Omega_{\left(A, M_{A}\right)}^{q}
$$

is surjective. Hence, every element on the right-hand side can be written nonuniquely as a differential $q$-form on $\left(W_{n}(A), M_{A}\right)$. The descending filtration of the de Rham-Witt complex by the differential graded ideals

$$
\mathrm{Fil}^{s} W_{n} \Omega_{\left(A, M_{A}\right)}^{*}=V^{s} W_{n-s} \Omega_{\left(A, M_{A}\right)}^{*}+d V^{s} W_{n-s} \Omega_{\left(A, M_{A}\right)}^{*}
$$

is called the standard filtration. It satisfies

$$
\begin{aligned}
& F\left(\mathrm{Fil}^{s} W_{n} \Omega_{\left(A, M_{A}\right)}^{q}\right) \subset \mathrm{Fil}^{s-1} W_{n-1} \Omega_{\left(A, M_{A}\right)}^{q}, \\
& V\left(\mathrm{Fil}^{s} W_{n} \Omega_{\left(A, M_{A}\right)}^{q}\right) \subset \mathrm{Fil}^{s+1} W_{n+1} \Omega_{\left(A, M_{A}\right)}^{q}
\end{aligned}
$$

but, in general, it is not multiplicative. The restriction induces an isomorphism

$$
W_{n} \Omega_{\left(A, M_{A}\right)}^{q} / \mathrm{Fil}^{s} W_{n} \Omega_{\left(A, M_{A}\right)}^{q} \stackrel{\sim}{\longrightarrow} W_{s} \Omega_{\left(A, M_{A}\right)}^{q} .
$$

1.2. Let $X$ be as in the introduction. We recall from [16] that the canonical $\log$-structure on $X$ is given by the cartesian square of sheaves of monoids

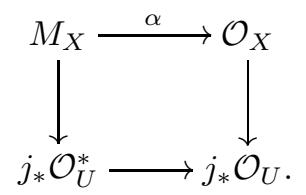

A choice of uniformizer $\pi$ of $V$ gives rise to an isomorphism

$$
\mathcal{O}_{X}^{*} \times \mathbb{N}_{0} \stackrel{\sim}{\longrightarrow} M_{X}
$$

that takes $(u, i)$ to $\pi^{i} u$. In this case, the de Rham-Witt complex

$$
W \cdot \Omega_{\left(X, M_{X}\right)}^{*}=W \cdot \Omega_{\left(\mathcal{O}_{X}, M_{X}\right)}^{*}
$$

has an additional filtration by the differential graded ideals

$$
\operatorname{Fil}_{U}^{m} W_{n} \Omega_{\left(X, M_{X}\right)}^{*}
$$


generated by $W_{n}\left(\mathfrak{m}^{j} \mathcal{O}_{X}\right)$, if $m=2 j$ is even, and by $W_{n}\left(\mathfrak{m}^{j} \mathcal{O}_{X}\right) \cdot d \log _{n} M_{X}$ and $W_{n}\left(\mathfrak{m}^{j+1} \mathcal{O}_{X}\right)$, if $m=2 j+1$ is odd. Here $\mathfrak{m}$ is the maximal ideal of $V$. We call this filtration the $U$-filtration.

Lemma 1.2.1. The $U$-filtration is multiplicative and is preserved by the restriction, Frobenius, and Verschiebung maps. Moreover, if $X_{j}=X \times_{\operatorname{Spec} V} \operatorname{Spec}\left(V / \mathfrak{m}^{j}\right)$, if $i_{j}: X_{j} \rightarrow X$ is the closed immersion, and if $\alpha: M_{X_{j}} \rightarrow \mathcal{O}_{X_{j}}$ is the induced pre-log structure, then the canonical projection induces an isomorphism

$$
i_{j}^{*} W_{n} \Omega_{\left(X, M_{X}\right)}^{q} / i_{j}^{*} \mathrm{Fil}_{U}^{2 j} W_{n} \Omega_{\left(X, M_{X}\right)}^{q} \stackrel{\sim}{\longrightarrow} W_{n} \Omega_{\left(X_{j}, M_{X_{j}}\right.}^{q} .
$$

Proof. A functor which has a right adjoint preserves initial objects. Hence

$$
i_{j}^{*} W_{n} \Omega_{\left(X, M_{X}\right)}^{q} / i_{j}^{*} \operatorname{Fil}_{U}^{2 j} W_{n} \Omega_{\left(X, M_{X}\right)}^{q}=W_{n} \Omega_{\left(i_{j}^{*} \mathcal{O}_{X}, i_{j}^{*} M_{X}\right)}^{q} / \mathrm{Fil}_{U}^{2 j} W_{n} \Omega_{\left(i_{j}^{*} \mathcal{O}_{X}, i_{j}^{*} M_{X}\right)}^{q} .
$$

Let $(B, M)$ be a log-ring, let $J \subset B$ be an ideal, and let $(\bar{B}, \bar{M})$ be the ring $\bar{B}=B / J$ with the induced pre-log-structure given by the composition

$$
\bar{\alpha}: \bar{M}=M \stackrel{\alpha}{\rightarrow} B \stackrel{\mathrm{pr}}{\longrightarrow} B / J .
$$

One shows as in [6, Lemma 2.4] that the canonical projection induces a surjection

$$
W_{n} \Omega_{(B, M)}^{*} \rightarrow W_{n} \Omega_{(\bar{B}, \bar{M})}^{*}
$$

and that the kernel is equal to the differential graded ideal generated by the ideal $W_{n}(J) \subset W_{n}(B)$. The lemma is a special case of this statement.

Lemma 1.2.2. Let $e$ be the ramification index of $V$ and $e^{\prime \prime}=e /(p-1)$. Then

$$
\begin{array}{ll}
p \mathrm{Fil}_{U}^{2 j} W_{n} \Omega_{\left(X, M_{X}\right)}^{q} \subset \mathrm{Fil}_{U}^{2 \min \{j+e, p j\}} W_{n} \Omega_{\left(X, M_{X}\right)}^{q}, & \text { for } j \geqslant 0, \\
p \mathrm{Fil}_{U}^{2 j} W_{n} \Omega_{\left(X, M_{X}\right)}^{q}=\operatorname{Fil}_{U}^{2(j+e)} W_{n} \Omega_{\left(X, M_{X}\right)}^{q}, & \text { for } j \geqslant e^{\prime \prime} .
\end{array}
$$

Proof. By the definition of the $U$-filtration, it suffices to show that

$$
\begin{aligned}
p W_{n}\left(\mathfrak{m}^{j} \mathcal{O}_{X}\right) & \subset W_{n}\left(\mathfrak{m}^{\min \{j+e, p j\}} \mathcal{O}_{X}\right), & & \text { for } j \geqslant 0, \\
W_{n}\left(\mathfrak{m}^{j+e} \mathcal{O}_{X}\right) & \subset p W_{n}\left(\mathfrak{m}^{j} \mathcal{O}_{X}\right), & & \text { for } j \geqslant e^{\prime \prime} .
\end{aligned}
$$

Let $\pi$ be a uniformizer of $V$ with minimal polynomial $x^{e}+p \theta(x)$ and recall from the proof of [10, Prop. 3.1.5] that $[\pi]^{e}+\theta([\pi]) V(1)$ is contained in $p W_{n}\left(\mathcal{O}_{X}\right)$. The second inclusion follows by iterated use of this congruence. Finally, we recall from the proof of [10, Lemma 3.1.1] that $p$ is congruent to $[p]+V(1)$ modulo $p V W_{n}\left(\mathcal{O}_{X}\right)$. The first inclusion follows by induction, since $p$ has valuation $e$.

The map $d \log _{n}: M_{X} \rightarrow W_{n} \Omega_{\left(X, M_{X}\right)}^{1}$ gives rise to a map of graded rings

$$
T_{\mathbb{Z}}\left(M_{X}^{\mathrm{gp}}\right) \rightarrow W_{n} \Omega_{\left(X, M_{X}\right)}^{*}
$$

from the tensor algebra of the group completion of the monoid $M_{X}$. There is a descending filtration of the left-hand side by graded ideals

$$
\operatorname{Fil}_{U}^{m} T_{\mathbb{Z}}\left(M_{X}^{\mathrm{gp}}\right),
$$

which corresponds to the $U$-filtration on the right-hand side. To define it, we first choose a uniformizer $\pi$ of $V$ such that we have the isomorphism

$$
\mathcal{O}_{X}^{*} \times \mathbb{Z} \stackrel{\sim}{\longrightarrow} M_{X}^{\text {gp }}
$$

that takes $(u, i)$ to $\pi^{i} u$. We define $\operatorname{Fil}_{U}^{m} T_{\mathbb{Z}}\left(M_{X}^{\mathrm{gp}}\right)$ to be $T_{\mathbb{Z}}\left(M_{X}^{\mathrm{gp}}\right)$, if $m=0$, and to be the graded ideal generated by $\left(1+\mathfrak{m}^{j} \mathcal{O}_{X}\right) \times\{0\} \subset M_{X}^{\text {gp }}$, if $m=2 j$ and $j>0$, by 
$\left(1+\mathfrak{m} \mathcal{O}_{X}\right) \times \mathbb{Z} \subset M_{X}^{\mathrm{gp}}$, if $m=1$, and by $\left(1+\mathfrak{m}^{j} \mathcal{O}_{X}\right) \times\{0\} \otimes\{1\} \times \mathbb{Z} \subset M_{X}^{\mathrm{gp}} \otimes M_{X}^{\mathrm{gp}}$ and $\left(1+\mathfrak{m}^{j+1} \mathcal{O}_{X}\right) \times\{0\} \subset M_{X}^{g p}$, if $m=2 j+1$ and $j>1$.

Lemma 1.2.3. Let $x$ be a local section of $\mathfrak{m}^{j} \mathcal{O}_{X}$. Then

$$
d \log _{n}(1+x) \equiv \sum_{0 \leqslant s<n} d V^{s}\left([x]_{n-s}\right)
$$

modulo $\operatorname{Fil}_{U}^{4 j} W_{n} \Omega_{\left(X, M_{X}\right)}^{1}$.

Proof. We first show that if $R$ is a ring and $x \in R$, then

$$
[1+x]_{n}-[1]_{n} \equiv \sum_{0 \leqslant s<n} V^{s}\left([x]_{n-s}\right)
$$

modulo the ideal $W_{n}\left(\left(x^{2}\right)\right) \subset W_{n}(R)$. By naturality, we may assume that $R=\mathbb{Z}[x]$. If we write $[1+x]_{n}-[1]_{n}=\left(a_{0}, a_{1}, \ldots, a_{n-1}\right)$, then the statement we wish to show is that $a_{s} \equiv x$ modulo $\left(x^{2}\right)$, for all $0 \leqslant s<n$. The statement for $s=0$ is clear. We consider the ghost coordinate

$$
(1+x)^{p^{s}}-1=a_{0}^{p^{s}}+p a_{1}^{p^{s-1}}+\cdots+p^{s-1} a_{s-1}^{p}+p^{s} a_{s} .
$$

The left-hand side is equivalent to $p^{s} x$ modulo $\left(x^{2}\right)$, and the right-hand side, inductively, is equivalent to $p^{s} a_{s}$ modulo $\left(x^{2}\right)$. It follows that $a_{s}$ is equivalent to $x$ modulo $\left(x^{2}\right)$ as desired. If $x$ is a local section of $\mathfrak{m}^{j} \mathcal{O}_{X}$, we may conclude that

$$
[1+x]_{n}-[1]_{n} \equiv \sum_{0 \leqslant s<n} V^{s}\left([x]_{n-s}\right)
$$

modulo $W_{n}\left(\mathfrak{m}^{2 j} \mathcal{O}_{X}\right)$. Differentiating this congruence we find that

$$
d\left([1+x]_{n}\right) \equiv \sum_{0 \leqslant s<n} d V^{s}\left([x]_{n-s}\right)
$$

modulo $\mathrm{Fil}_{U}^{4 j} W_{n} \Omega_{\left(X, M_{X}\right)}^{1}$. It remains to show that the left-hand side is congruent to $d \log _{n}(1+x)$ modulo $\mathrm{Fil}_{U}^{4 j} W_{n} \Omega_{\left(X, M_{X}\right)}^{1}$. By definition, we have

$$
[1+x]_{n} d \log _{n}(1+x)=d\left([1+x]_{n}\right),
$$

and $[1+x]_{n}$ is a unit in $W_{n}\left(\mathcal{O}_{X} / \mathfrak{m}^{2 j} \mathcal{O}_{X}\right)$. Therefore, it will suffice to show that the product $\left([1+x]_{n}-[1]_{n}\right) d\left([1+x]_{n}\right)$ is congruent to zero modulo $\mathrm{Fil}_{U}^{4 j} W_{n} \Omega_{\left(X, M_{X}\right)}^{1}$. But this is so, since the two factors lie in $\operatorname{Fil}_{U}^{2 j} W_{n} \Omega_{\left(X, M_{X}\right)}^{*}$ and since the $U$-filtration is multiplicative.

Lemma 1.2 .3 determines the value of the map $d \log _{n}$ modulo higher filtration on $\mathrm{Fil}_{U}^{m} T_{\mathbb{Z}}\left(M_{X}^{\mathrm{gp}}\right)$, for $m \geqslant 2$. Moreover, there is a commutative square

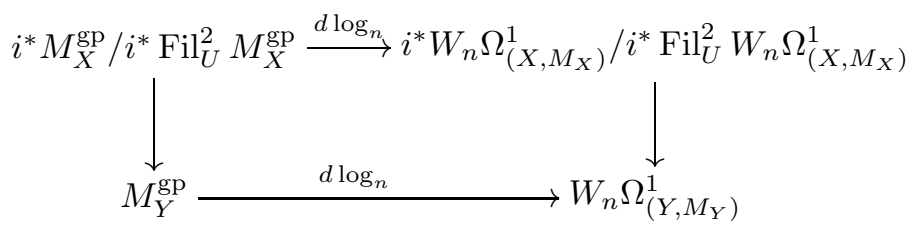

where the right-hand vertical map is an isomorphism by an argument similar to the proof of Lemma 1.2.1. 
1.3. We recall from [11, Lemma 7.1.2] that the sheaf

$$
\bar{W}_{n} \Omega_{\left(X, M_{X}\right)}^{q}=W_{n} \Omega_{\left(X, M_{X}\right)}^{q} / p W_{n} \Omega_{\left(X, M_{X}\right)}^{q}
$$

is a quasi-coherent sheaf of $\bar{W}_{n}\left(\mathcal{O}_{X}\right)$-modules on the small étale site of $X$. Since this sheaf is supported on $Y$, we may as well consider the sheaf

$$
E_{n}^{q}=i^{*} \bar{W}_{n} \Omega_{\left(X, M_{X}\right)}^{q}
$$

of quasi-coherent $i^{*} \bar{W}_{n}\left(\mathcal{O}_{X}\right)$-modules on $Y$. We show that, Zariski locally, the sheaf $E_{n}^{q}$ has a non-canonical structure of a quasi-coherent $\mathcal{O}_{Y}$-module. Let $\varphi$ be the absolute Frobenius on $Y$.

Lemma 1.3.1. Let $x_{1}, \ldots, x_{r}$ be local coordinates of an open neighborhood in $X$ of a point of $Y$, and let $\bar{x}_{1}, \ldots, \bar{x}_{r}$ be the corresponding local coordinates on $Y$. Then there is, in the corresponding open neighborhood of $Y$, a strict map of pro-rings

$$
\delta: \mathcal{O}_{Y} \rightarrow i^{*} \bar{W} \cdot\left(\mathcal{O}_{X}\right)
$$

such that $\delta_{n}\left(\bar{x}_{i}\right)=\left[x_{i}\right]_{n}, 1 \leqslant i \leqslant r$, and such that $F \delta_{n}=\delta_{n-1} \varphi$.

Proof. The ring homomorphism

$$
f: W(k)\left[x_{1}, \ldots, x_{r}\right] \rightarrow W(k)\left[x_{1}, \ldots, x_{r}\right]
$$

given by the Frobenius on $W(k)$ and by $f\left(x_{i}\right)=x_{i}^{p}, 1 \leqslant i \leqslant r$, is a lifting of the Frobenius on $k\left[\bar{x}_{1}, \ldots, \bar{x}_{r}\right]$. It determines a ring homomorphism

$$
s_{f}: W(k)\left[x_{1}, \ldots, x_{r}\right] \rightarrow W_{n}\left(W(k)\left[x_{1}, \ldots, x_{r}\right]\right)
$$

that is characterized by $w_{j} s_{f}=f^{j}, 0 \leqslant j<n$, and, after reduction modulo $p$, a ring homomorphism

$$
\bar{s}_{f}: k\left[\bar{x}_{1}, \ldots, \bar{x}_{r}\right] \rightarrow \bar{W}_{n}\left(W(k)\left[x_{1}, \ldots, x_{r}\right]\right) .
$$

We compose this map with the ring homomorphisms

$$
\bar{W}_{n}\left(W(k)\left[x_{1}, \ldots, x_{r}\right]\right) \rightarrow \bar{W}_{n}\left(V\left[x_{1}, \ldots, x_{r}\right]\right) \rightarrow \bar{W}_{n}\left(\mathcal{O}_{X}\right)
$$

induced from the unique ring homomorphism $W(k) \rightarrow V$ that induces the identity on residue fields and the chosen ring homomorphism $g: V\left[x_{1}, \ldots, x_{r}\right] \rightarrow \mathcal{O}_{X}$ to get the top horizontal map in the following diagram:

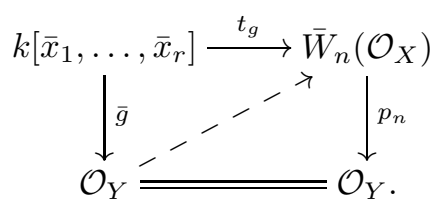

The right-hand vertical map is the composition of the restriction of Witt vectors with kernel $V \bar{W}_{n}\left(\mathcal{O}_{X}\right)$ and the canonical projection $\overline{\mathcal{O}}_{X} \rightarrow \mathcal{O}_{Y}$ with kernel $\mathfrak{m} \mathcal{O}_{X} / p \mathcal{O}_{X}$. Since both ideals are nilpotent, and since the left-hand vertical map is étale, there exists a unique ring homomorphism

$$
\delta_{n}: \mathcal{O}_{Y} \rightarrow \bar{W}_{n}\left(\mathcal{O}_{X}\right)
$$

making the above diagram commute. Moreover, one immediately verifies that $R \delta_{n}=\delta_{n-1}$ and that $\delta_{n}\left(\bar{x}_{i}\right)=\left[x_{i}\right]_{n}, 1 \leqslant i \leqslant r$, as stated. It remains to show 
that $F \delta_{n}=\delta_{n-1} \varphi$, or equivalently, that the right-hand square in the following diagram commutes:

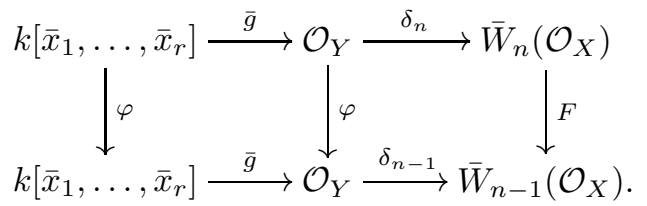

Since the outer square commutes, and since the left-hand square is cocartesian, it follows that there exists a map $\delta_{n-1}^{\prime}: \mathcal{O}_{Y} \rightarrow \bar{W}_{n-1}\left(\mathcal{O}_{X}\right)$ that makes the right-hand square commute. To show that $\delta_{n-1}^{\prime}=\delta_{n-1}$, it will suffice to show that

$$
\mathcal{O}_{Y} \stackrel{\delta_{n-1}^{\prime}}{\longrightarrow} \bar{W}_{n-1}\left(\mathcal{O}_{X}\right) \stackrel{p_{n-1}}{\longrightarrow} \mathcal{O}_{Y}
$$

is the identity map. This, in turn, follows from the calculation

$$
p_{n-1} F \delta_{n} \bar{g}=\varphi p_{n} \delta_{n} \bar{g}=\varphi \bar{g},
$$

since the left-hand square of the diagram above is cocartesian.

Proposition 1.3.2. The sheaf $E_{n}^{q}=i^{*} \bar{W}_{n} \Omega_{\left(X, M_{X}\right)}^{q}$ has, Zariski locally on $Y$, the structure of a free $\mathcal{O}_{Y}$-module of rank

$$
\operatorname{rk}_{\mathcal{O}_{Y}} E_{n}^{q}=\left(\begin{array}{c}
r+1 \\
q
\end{array}\right) e \cdot \sum_{s=0}^{n-1} p^{r s} .
$$

Proof. We consider the sheaf $E_{n}^{q}$ with the Zariski locally defined $\mathcal{O}_{Y}$-module structure given by Lemma 1.3.1. The statement of the proposition is unchanged by étale extensions, so we may assume that $X=\mathbb{A}_{V}^{r}$. We prove the proposition in this case by induction on $r$. The basic case $r=0$ follows from [10, Prop. 3.4.1]. In the induction step we use [11, Thm. B], which shows that the $\mathcal{O}_{\mathbb{A}_{k}^{r}}$-module $i^{*} \bar{W}_{n} \Omega_{\left(\mathbb{A}_{V}^{r}, M_{\mathbb{A}_{V}^{r}}\right)}$ is the base-change along $\mathbb{A}_{k}^{r-1} \hookrightarrow \mathbb{A}_{k}^{r}$ of the $\mathcal{O}_{\mathbb{A}_{k}^{r-1}}$-module

$$
\begin{aligned}
& i^{*} \bar{W}_{n} \Omega_{\left(\mathbb{A}_{V}^{r-1}, M_{\mathbb{A}_{V}^{r-1}}^{q}\right)}^{q} \oplus \bigoplus_{s=1}^{n-1} \bigoplus_{0 \leqslant j<p^{s}} F_{*}^{s}\left(i^{*} \bar{W}_{n-s} \Omega_{\left(\mathbb{A}_{V}^{r-1}, M_{\mathbb{A}_{V}^{r-1}}\right)}^{q}\right) \\
& \oplus i^{*} \bar{W}_{n} \Omega_{\left(\mathbb{A}_{V}^{r-1}, M_{\mathbb{A}_{V}^{r-1}}^{q-1}\right.} \oplus \bigoplus_{s=1}^{n-1} \bigoplus_{0 \leqslant j<p^{s}} F_{*}^{s}\left(i^{*} \bar{W}_{n-s} \Omega_{\left(\mathbb{A}_{V}^{r-1}, M_{\mathbb{A}_{V}^{r-1}}\right)}^{q-1}\right),
\end{aligned}
$$

where the index $0 \leqslant j<p^{s}$ is required to not be divisible by $p$. By induction and by Lemma 1.3.1, this module has rank

$$
\begin{aligned}
& \left(\begin{array}{l}
r \\
q
\end{array}\right) \cdot e \cdot\left(\sum_{t=0}^{n-1} p^{(r-1) t}+\sum_{s=1}^{n-1}\left(p^{s}-p^{s-1}\right) p^{(r-1) s} \sum_{t=0}^{n-1-s} p^{(r-1) t}\right) \\
& +\left(\begin{array}{c}
r \\
q-1
\end{array}\right) \cdot e \cdot\left(\sum_{t=0}^{n-1} p^{(r-1) t}+\sum_{s=1}^{n-1}\left(p^{s}-p^{s-1}\right) p^{(r-1) s} \sum_{t=0}^{n-1-s} p^{(r-1) t}\right),
\end{aligned}
$$

and since

$$
\left(\begin{array}{c}
r \\
q-1
\end{array}\right) \cdot e+\left(\begin{array}{l}
r \\
q
\end{array}\right) \cdot e=\left(\begin{array}{c}
r+1 \\
q
\end{array}\right) \cdot e
$$


it remains to show that

$$
\sum_{t=0}^{n-1} p^{(r-1) t}+\sum_{s=1}^{n-1}\left(p^{s}-p^{s-1}\right) p^{(r-1) s} \sum_{t=0}^{n-1-s} p^{(r-1) t}=\sum_{s=0}^{n-1} p^{r s} .
$$

To see this, we rewrite the first summand on the left-hand side as

$$
1+p^{r-1} \sum_{t=0}^{n-2} p^{(r-1) t}
$$

and rewrite the $s$ th summand on the left-hand side as

$$
p^{r s}+p^{r(s+1)-1} \sum_{t=0}^{n-(s+1)-1} p^{(r-1) t}-p^{r s-1} \sum_{t=0}^{n-s-1} p^{(r-1) t} .
$$

The statement follows.

Definition 1.3.3. Two local sections $\omega$ and $\omega^{\prime}$ of $E_{n}^{q}$ are similar if there exists a unit $u \in k^{*}$ such that whenever both $\omega$ and $\omega^{\prime}$ are local sections of $\mathrm{Fil}_{U}^{m} E_{n}^{q}$, then $\omega-u \cdot \omega^{\prime}$ is a local section of $\mathrm{Fil}_{U}^{m+1} E_{n}^{q}$. We write $\omega \doteq \omega^{\prime}$ if $\omega$ and $\omega^{\prime}$ are similar.

We note that if $\omega$ and $\omega^{\prime}$ are similar, then so are $R(\omega)$ and $R\left(\omega^{\prime}\right), F(\omega)$ and $F\left(\omega^{\prime}\right), d(\omega)$ and $d\left(\omega^{\prime}\right)$, and $V(\omega)$ and $V\left(\omega^{\prime}\right)$. Let $\pi$ be a uniformizer of $V$. Then by the proof of [10, Prop. 3.1.5], the following similarity holds in the $\operatorname{ring} E_{n}^{0}$ :

$$
[\pi]_{n}^{e} \doteq V(1) \text {. }
$$

In the statements and proofs of Prop. 1.3.4 and Thm.1.3.5 below we shall abbreviate $[x]=[x]_{n}$ and $d \log x=d \log _{n} x$.

Proposition 1.3.4. Let $x_{1}, \ldots, x_{r}$ be local coordinates of an open neighborhood of $X$ around a point of $Y$. Then in the corresponding open neighborhood of $Y$ the sheaf $E_{n}^{q}=i^{*} \bar{W}_{n} \Omega_{\left(X, M_{X}\right)}^{q}$ is a free $\mathcal{O}_{Y}$-module with a basis given as follows. Let $0 \leqslant s<n$, let $0 \leqslant i_{1}, \ldots, i_{r}<p^{s}$ and let $0 \leqslant j<e$.

(i) If not all $i_{m}, 1 \leqslant m \leqslant r$, are zero, let $v=\min \left\{v_{p}\left(i_{1}\right), \ldots, v_{p}\left(i_{r}\right), v_{p}\left(j-e^{\prime}\right)\right\}$; if $v<v_{p}\left(i_{m}\right)$, for all $1 \leqslant m \leqslant r$, then the local sections

$$
\begin{aligned}
& V^{s}\left(\left[x_{1}\right]^{i_{1}} \ldots\left[x_{r}\right]^{i_{r}}[\pi]^{j} d \log x_{m_{1}} \ldots d \log x_{m_{q}}\right), \\
& d V^{s}\left(\left[x_{1}\right]^{i_{1}} \ldots\left[x_{r}\right]^{i_{r}}[\pi]^{j} d \log x_{m_{1}} \ldots d \log x_{m_{q-1}}\right),
\end{aligned}
$$

where $1 \leqslant m_{1}<\cdots<m_{q} \leqslant r$ (resp. $1 \leqslant m_{1}<\cdots<m_{q-1} \leqslant r$ ), form a basis. If $v=v_{p}\left(i_{m}\right)$, for some $1 \leqslant m \leqslant r$, let $m$ be maximal with this property; then the local sections

$$
\begin{aligned}
& V^{s}\left(\left[x_{1}\right]^{i_{1}} \ldots\left[x_{r}\right]^{i_{r}}[\pi]^{j} d \log x_{m_{1}} \ldots d \log x_{m_{q}}\right), \\
& V^{s}\left(\left[x_{1}\right]^{i_{1}} \ldots\left[x_{r}\right]^{i_{r}}[\pi]^{j} d \log x_{m_{1}} \ldots d \log x_{m_{q-1}} d \log \pi\right), \\
& d V^{s}\left(\left[x_{1}\right]^{i_{1}} \ldots\left[x_{r}\right]^{i_{r}}[\pi]^{j} d \log x_{m_{1}} \ldots d \log x_{m_{q-1}}\right), \\
& d V^{s}\left(\left[x_{1}\right]^{i_{1}} \ldots\left[x_{r}\right]^{i_{r}}[\pi]^{j} d \log x_{m_{1}} \ldots d \log x_{m_{q-2}} d \log \pi\right),
\end{aligned}
$$

where $1 \leqslant m_{1}<\cdots<m_{q} \leqslant r$ (resp. $1 \leqslant m_{1}<\cdots<m_{q-1} \leqslant r$, resp. $1 \leqslant m_{1}<$ $\left.\cdots<m_{q-2} \leqslant r\right)$, and where all $m_{i} \neq m$ form a basis.

(ii) If all $i_{m}, 1 \leqslant m \leqslant r$, are zero, the local sections

$$
V^{s}\left([\pi]^{j} d \log x_{m_{1}} \ldots d \log x_{m_{q}}\right)
$$


where $1 \leqslant m_{1}<\cdots<m_{q} \leqslant r$, and if $s>v_{p}\left(j-e^{\prime}\right)$, the local sections

$$
d V^{s}\left([\pi]^{j} d \log x_{m_{1}} \ldots d \log x_{m_{q-1}}\right)
$$

where $1 \leqslant m_{1}<\cdots<m_{q-1} \leqslant r$, and if $s \leqslant v_{p}\left(j-e^{\prime}\right)$, the local sections

$$
V^{s}\left([\pi]^{j} d \log x_{m_{1}} \ldots d \log x_{m_{q-1}} d \log \pi\right)
$$

where $1 \leqslant m_{1}<\cdots<m_{q-1} \leqslant r$, form a basis.

Proof. Let $\Gamma_{n}^{q}$ be the set of local sections of $E_{n}^{q}$ listed in the statement. It is clear that the cardinality of this set is equal to

$$
\left(\left(\begin{array}{l}
r \\
q
\end{array}\right)+\left(\begin{array}{c}
r \\
q-1
\end{array}\right)\right) e \cdot \sum_{s=0}^{n-1} p^{r s}=\left(\begin{array}{c}
r+1 \\
q
\end{array}\right) e \cdot \sum_{s=1}^{n-1} p^{r s} .
$$

Hence, by Prop. 1.3.2, it suffices to show that $\Gamma_{n}^{q}$ generates $E_{n}^{q}$ as an $\mathcal{O}_{Y}$-module. To do this, we first show that a larger set $\Gamma_{n}^{q \prime}$ of local sections generates $E_{n}^{q}$ as an $\mathcal{O}_{Y}$-module and then show that the elements of the complement $\Gamma_{n}^{q \prime} \backslash \Gamma_{n}^{q}$ can be expressed as $\mathcal{O}_{Y}$-linear combinations of elements of $\Gamma_{n}^{q}$. It will suffice to show that the elements of $\Gamma_{n}^{q \prime} \backslash \Gamma_{n}^{q}$ are similar in the sense of Def. 1.3.3 to $\mathcal{O}_{Y}$-linear combinations of the elements of $\Gamma_{n}^{q}$. Indeed, the $U$-filtration of $E_{n}^{q}$ is finite.

We define $\Gamma_{n}^{q \prime}$ to be the set consisting of the local sections

$$
\begin{aligned}
& V^{s}\left(\left[x_{1}\right]^{i_{1}} \ldots\left[x_{r}\right]^{i_{r}}[\pi]^{j} d \log x_{m_{1}} \ldots d \log x_{m_{q}}\right), \\
& d V^{s}\left(\left[x_{1}\right]^{i_{1}} \ldots\left[x_{r}\right]^{i_{r}}[\pi]^{j} d \log x_{m_{1}} \ldots d \log x_{m_{q-1}}\right), \\
& V^{s}\left(\left[x_{1}\right]^{i_{1}} \ldots\left[x_{r}\right]^{i_{r}}[\pi]^{j} d \log x_{m_{1}} \ldots d \log x_{m_{q-1}} d \log \pi\right), \\
& d V^{s}\left(\left[x_{1}\right]^{i_{1}} \ldots\left[x_{r}\right]^{i_{r}}[\pi]^{j} d \log x_{m_{1}} \ldots d \log x_{m_{q-2}} d \log \pi\right),
\end{aligned}
$$

where $0 \leqslant s<n$, where $0 \leqslant i_{1}, \ldots, i_{r}<p^{s}$, where $0 \leqslant j<e$, and where $1 \leqslant m_{1}<$ $\cdots<m_{q} \leqslant r$ (resp. $1 \leqslant m_{1}<\cdots<m_{q-1} \leqslant r$, resp. $1 \leqslant m_{1}<\cdots<m_{q-2} \leqslant r$ ). We also consider the larger set $\Gamma_{n}^{q \prime \prime}$ of local sections defined similarly except that the indices $i_{1}, \ldots, i_{r}$ are allowed to take all non-negative integer values. We claim that the set $\Gamma_{n}^{q \prime \prime}$ generates $E_{n}^{q}$ as a sheaf of $k$-vector spaces. Indeed, the case $n=1$ is standard and the general case follows by an induction argument based on the exact sequence

$$
\mathrm{Fil}^{n-1} E_{n}^{q} \rightarrow E_{n}^{q} \stackrel{R}{\longrightarrow} E_{n-1}^{q} \rightarrow 0
$$

and the surjective map

$$
V^{n-1}+d V^{n-1}: E_{1}^{q} \oplus E_{1}^{q-1} \rightarrow \mathrm{Fil}^{n-1} E_{n}^{q}
$$

Here the latter map is a $k$-linear map since $k$ is a perfect field. Next, iterated use of the relations

$$
\begin{aligned}
V^{s}\left(\left[x_{m}\right]^{p^{s}} \cdot \omega\right) & =\left[x_{m}\right] \cdot V^{s}(\omega), \\
d V^{s}\left(\left[x_{m}\right]^{p^{s}} \cdot \omega\right) & =\left[x_{m}\right] \cdot d V^{s}(\omega)+\left[x_{m}\right] \cdot V^{s}\left(d \log x_{m} \cdot \omega\right)
\end{aligned}
$$

shows that the local sections in $\Gamma_{n}^{q \prime \prime} \backslash \Gamma_{n}^{q \prime}$ can be expressed as $\mathcal{O}_{Y}$-linear combinations of the local sections in $\Gamma_{n}^{q \prime}$. Hence $\Gamma_{n}^{q \prime}$ generates $E_{n}^{q}$ as an $\mathcal{O}_{Y}$-module. We proceed to show that the elements of $\Gamma_{n}^{q^{\prime}} \backslash \Gamma_{n}^{q}$ are similar to $\mathcal{O}_{Y}$-linear combinations of elements of $\Gamma_{n}^{q}$ by considering several cases. 
First, let $0<s<n$, let $0 \leqslant i_{1}, \ldots, i_{r}<p^{s}$, and let $0 \leqslant j<e$. We assume that not all $i_{m}$ are zero and that all $i_{m}$ satisfy $v_{p}\left(i_{m}\right)>v_{p}\left(j-e^{\prime}\right)=v$. Then the local sections of the form

$$
\begin{aligned}
& V^{s}\left(\left[x_{1}\right]^{i_{r}} \ldots\left[x_{r}\right]^{i_{r}} d \log x_{m_{1}} \ldots d \log x_{m_{q-1}} d \log \pi\right), \\
& d V^{s}\left(\left[x_{1}\right]^{i_{r}} \ldots\left[x_{r}\right]^{i_{r}} d \log x_{m_{1}} \ldots d \log x_{m_{q-2}} d \log \pi\right),
\end{aligned}
$$

where $1 \leqslant m_{1}<\cdots<m_{q-1} \leqslant r$ (resp. $1 \leqslant m_{1}<\cdots<m_{q-2} \leqslant r$ ), are elements of $\Gamma_{n}^{q \prime} \backslash \Gamma_{n}^{q}$. We will argue that the top elements are similar to $\mathcal{O}_{Y}$-linear combinations of elements of $\Gamma_{n}^{q}$; the bottom elements are treated analogously. We note that $0 \leqslant v<s$ and define $s^{\prime}=s-v$. We also define $i_{m}^{\prime}=p^{-v} i_{m}, 1 \leqslant m \leqslant r$, and

$$
j^{\prime}=p^{-v} j+e\left(\frac{p^{-v}-1}{p^{-1}-1}\right)=p^{-v}\left(j+p e\left(\frac{p^{v}-1}{p-1}\right)\right) .
$$

Then iterated use of the similarity $[\pi]^{e} \doteq V(1)$ shows that

$$
\begin{aligned}
& V^{s}\left(\left[x_{1}\right]^{i_{1}} \ldots\left[x_{r}\right]^{i_{r}}[\pi]^{j} d \log x_{m_{1}} \ldots d \log x_{m_{q-1}} d \log \pi\right) \\
& \doteq V^{s^{\prime}}\left(\left[x_{1}\right]^{i_{1}^{\prime}} \ldots\left[x_{r}\right]^{i_{r}^{\prime}}[\pi]^{j^{\prime}} d \log x_{m_{1}} \ldots d \log x_{m_{q-1}} d \log \pi\right) .
\end{aligned}
$$

The integers $i_{m}^{\prime}, 1 \leqslant m \leqslant r$, are all divisible by $p$, and the integer $j^{\prime}$ is not divisible by $p$. Therefore, since $d$ is a derivation, we conclude that

$$
\begin{aligned}
& V^{s^{\prime}}\left(\left[x_{1}\right]^{i_{1}^{\prime}} \ldots\left[x_{r}\right]^{i_{r}^{\prime}}[\pi]^{j^{\prime}} d \log x_{m_{1}} \ldots d \log x_{m_{q-1}} d \log \pi\right) \\
& \doteq V^{s^{\prime}} d\left(\left[x_{1}\right]^{i_{1}^{\prime}} \ldots\left[x_{r}\right]^{i_{r}^{\prime}}[\pi]^{j^{\prime}} d \log x_{m_{1}} \ldots d \log x_{m_{q-1}}\right),
\end{aligned}
$$

which is equal to zero, since $s^{\prime}=s-v>0$ and $V d=p d V$.

We next let $0<s<n$, let $0 \leqslant i_{1}, \ldots, i_{r}<p^{s}$, and let $0 \leqslant j<e$, and assume that not all $i_{m}$ are zero. We further let $1 \leqslant m \leqslant r$ be maximal with the property that $v_{p}\left(i_{m}\right)=\min \left\{v_{p}\left(i_{1}\right), \ldots, v_{p}\left(i_{r}\right)\right\}$ and assume that $v=v_{p}\left(i_{m}\right) \leqslant v_{p}\left(j-e^{\prime}\right)$. Then the local sections

$$
\begin{aligned}
& V^{s}\left(\left[x_{1}\right]^{i_{1}} \ldots\left[x_{r}\right]^{i_{r}}[\pi]^{j} d \log x_{m_{1}} \ldots d \log x_{m_{q}}\right), \\
& V^{s}\left(\left[x_{1}\right]^{i_{1}} \ldots\left[x_{r}\right]^{i_{r}}[\pi]^{j} d \log x_{m_{1}} \ldots d \log x_{m_{q-1}} d \log \pi\right), \\
& d V^{s}\left(\left[x_{1}\right]^{i_{1}} \ldots\left[x_{r}\right]^{i_{r}}[\pi]^{j} d \log x_{m_{1}} \ldots d \log x_{m_{q-1}}\right), \\
& d V^{s}\left(\left[x_{1}\right]^{i_{1}} \ldots\left[x_{r}\right]^{i_{r}}[\pi]^{j} d \log x_{m_{1}} \ldots d \log x_{m_{q-2}} d \log \pi\right),
\end{aligned}
$$

where $1 \leqslant m_{1}<\cdots<m_{q} \leqslant r$ (resp. $1 \leqslant m_{1}<\cdots<m_{q-1} \leqslant r$, resp. $1 \leqslant m_{1}<$ $\left.\cdots<m_{q-2} \leqslant r\right)$, and where some $m_{i}=m$, are elements of $\Gamma_{n}^{q \prime} \backslash \Gamma_{n}^{q}$. We again argue that the top elements are similar to $\mathcal{O}_{Y}$-linear combinations of elements of $\Gamma_{n}^{q}$; the remaining elements are treated analogously. The integers $v$ satisfy $0 \leqslant v<s$, and we define $s^{\prime}, i_{\ell}^{\prime}$, and $j^{\prime}$ as above such that we have the similarity

$$
\begin{aligned}
& V^{s}\left(\left[x_{1}\right]^{i_{1}} \ldots\left[x_{r}\right]^{i_{r}}[\pi]^{j} d \log x_{m_{1}} \ldots d \log x_{m_{q}}\right) \\
& \doteq V^{s^{\prime}}\left(\left[x_{1}\right]^{i_{1}^{\prime}} \ldots\left[x_{r}\right]^{i_{r}^{\prime}}[\pi]^{j^{\prime}} d \log x_{m_{1}} \ldots d \log x_{m_{q}}\right) .
\end{aligned}
$$

We claim that this local section is similar to the local section

$$
\begin{aligned}
& \sum_{\ell \neq m} i_{\ell}^{\prime} \cdot V^{s}\left(\left[x_{1}\right]^{i_{1}} \ldots\left[x_{r}\right]^{i_{r}}[\pi]^{j} d \log x_{\ell} d \log x_{m_{1}} \ldots \widehat{d \log x_{m}} \ldots d \log x_{m_{q}}\right) \\
& \quad+j^{\prime} \cdot V^{s}\left(\left[x_{1}\right]^{i_{1}} \ldots\left[x_{r}\right]^{i_{r}}[\pi]^{j} d \log \pi d \log x_{m_{1}} \ldots \widehat{\log x_{m}} \ldots d \log x_{m_{q}}\right)
\end{aligned}
$$


and hence similar to an $\mathcal{O}_{Y^{-}}$-linear combination of elements of $\Gamma_{n}^{q}$ as desired. Here the term $d \log x_{m}$ is omitted. Indeed, on the one hand, the local section

$$
V^{s^{\prime}} d\left(\left[x_{1}\right]^{i_{1}^{\prime}} \ldots\left[x_{r}\right]^{i_{r}^{\prime}}[\pi]^{j^{\prime}} d \log x_{m_{1}} \ldots \widehat{d \log x_{m}} \ldots d \log x_{m_{q}}\right)
$$

is equal to zero, since $s^{\prime}>0$ and $V d=p d V$, and on the other hand, this local section is equal to the sum

$$
\begin{aligned}
& \sum_{1 \leqslant \ell \leqslant r} i_{\ell}^{\prime} \cdot V^{s^{\prime}}\left(\left[x_{1}\right]^{i_{1}^{\prime}} \ldots\left[x_{r}\right]^{i_{r}^{\prime}}[\pi]^{j^{\prime}} d \log x_{\ell} d \log x_{m_{1}} \ldots \widehat{d \log x_{m}} \ldots d \log x_{m_{q}}\right) \\
& \quad+j^{\prime} \cdot V^{s^{\prime}}\left(\left[x_{1}\right]^{i_{1}^{\prime}} \ldots\left[x_{r}\right]^{i_{r}^{\prime}}[\pi]^{j^{\prime}} d \log \pi d \log x_{m_{1}} \ldots \widehat{d \log x_{m}} \ldots d \log x_{m_{q}}\right)
\end{aligned}
$$

since $d$ is a derivation. The individual summands in this sum are similar to the corresponding summands in the sum

$$
\begin{gathered}
\sum_{1 \leqslant \ell \leqslant r} i_{\ell}^{\prime} \cdot V^{s}\left(\left[x_{1}\right]^{i_{1}} \ldots\left[x_{r}\right]^{i_{r}}[\pi]^{j} d \log x_{\ell} d \log x_{m_{1}} \ldots \widehat{\log x_{m}} \ldots d \log x_{m_{q}}\right) \\
\quad+j^{\prime} \cdot V^{s}\left(\left[x_{1}\right]^{i_{1}} \ldots\left[x_{r}\right]^{i_{r}}[\pi]^{j} d \log \pi d \log x_{m_{1}} \ldots \widehat{d \log x_{m}} \ldots d \log x_{m_{q}}\right)
\end{gathered}
$$

from which the desired similarity follows since $i_{m}^{\prime}$ is not divisible by $p$.

Finally, let $0 \leqslant s<n$ and let $0 \leqslant j<e$. If $s \leqslant v_{p}\left(j-e^{\prime}\right)$, the local sections

$$
d V^{s}\left([\pi]^{j} d \log x_{m_{1}} \ldots d \log x_{m_{q-1}}\right),
$$

where $1 \leqslant m_{1}<\cdots<m_{q-1} \leqslant r$, and if $s>v_{p}\left(j-e^{\prime}\right)$, the local sections

$$
V^{s}\left([\pi]^{j} d \log x_{m_{1}} \ldots d \log x_{m_{q-1}} d \log \pi\right),
$$

where $1 \leqslant m_{1}<\cdots<m_{q-1} \leqslant r$, are elements of $\Gamma_{n}^{q \prime} \backslash \Gamma_{n}^{q}$. In the former case, let

$$
j^{\prime}=p^{-s} j+e\left(\frac{p^{-s}-1}{p^{-1}-1}\right)=p^{-s}\left(j+p e\left(\frac{p^{s}-1}{p-1}\right)\right) .
$$

We then have the similarity

$$
d V^{s}\left([\pi]^{j} d \log x_{m_{1}} \ldots d \log x_{m_{q-1}}\right) \doteq j^{\prime} \cdot V^{s}\left([\pi]^{j} d \log x_{m_{1}} \ldots d \log x_{m_{q-1}} d \log \pi\right),
$$

which shows that these elements are similar to $\mathcal{O}_{Y}$-linear combinations of elements of $\Gamma_{n}^{q}$. In the latter case, we let $v=v_{p}\left(j-e^{\prime}\right)$ and define $s^{\prime}=s-v$ and

$$
j^{\prime}=p^{-v} j+e\left(\frac{p^{-v}-1}{p^{-1}-1}\right)=p^{-v}\left(j+p e\left(\frac{p^{v}-1}{p-1}\right)\right) .
$$

Then $s^{\prime}>0$ and since $V d=p d V$ the similarity

$$
V^{s}\left([\pi]^{j} d \log x_{m_{1}} \ldots d \log x_{m_{q-1}} d \log \pi\right) \doteq V^{s^{\prime}} d\left([\pi]^{j^{\prime}} d \log x_{m_{1}} \ldots d \log x_{m_{q-1}}\right)
$$

shows that these elements of $\Gamma_{n}^{q \prime} \backslash \Gamma_{n}^{q}$ are similar to zero. This completes the proof of the proposition.

Theorem 1.3.5. For $0 \leqslant j<e^{\prime}$, let $v$ be the unique integer such that

$$
e\left(\frac{p^{-v}-1}{p^{-1}-1}\right) \leqslant j<e\left(\frac{p^{-(v+1)}-1}{p^{-1}-1}\right) .
$$

Let $x_{1}, \ldots, x_{r}$ be local coordinates of an open neighborhood on $X$ around a point of $Y$. Then, in the corresponding open neighborhood of $Y$, the sheaf

$$
E_{n}^{q}=i^{*} W_{n} \Omega_{\left(X, M_{X}\right)}^{q} / p i^{*} W_{n} \Omega_{\left(X, M_{X}\right)}^{q}
$$

has the following structure. 
(i) If $0 \leqslant v<n$, then $\operatorname{gr}_{U}^{2 j} E_{n}^{q}$ is a free $\mathcal{O}_{Y}$-module with a basis given as follows. If $p$ does not divide $j$ (resp. if $p$ divides $j$ ), let $0<s<n-v$, and let $0 \leqslant i_{1}, \ldots, i_{r}<p^{s}$ (resp. let $0 \leqslant i_{1}, \ldots, i_{r}<p^{s}$ not all divisible by $p$ ); let $1 \leqslant m \leqslant r$ be maximal with $i_{m}$ not divisible by $p$. Then the local sections

$$
\begin{aligned}
& V^{s}\left(\left[x_{1}\right]^{i_{1}} \ldots\left[x_{r}\right]^{i_{r}}[\pi]^{j} d \log x_{m_{1}} \ldots d \log x_{m_{q}}\right), \\
& d V^{s}\left(\left[x_{1}\right]^{i_{1}} \ldots\left[x_{r}\right]^{i_{r}}[\pi]^{j} d \log x_{m_{1}^{\prime}} \ldots d \log x_{m_{q-1}^{\prime}}\right),
\end{aligned}
$$

where $1 \leqslant m_{1}<\cdots<m_{q} \leqslant r$ and $1 \leqslant m_{1}^{\prime}<\cdots<m_{q-1}^{\prime} \leqslant r$, and where all $m_{i}$ and $m_{i}^{\prime}$ are different from $m$, together with the local sections

$$
[\pi]^{j} d \log x_{m_{1}} \ldots d \log x_{m_{q}},
$$

where $1 \leqslant m_{1}<\cdots<m_{q} \leqslant r$, form a basis.

(ii) If $0 \leqslant v<n$, then $\operatorname{gr}_{U}^{2 j+1} E_{n}^{q}$ is a free $\mathcal{O}_{Y}$-module with a basis given as follows. Let $0<s<n-v$, and let $0 \leqslant i_{1}, \ldots, i_{r}<p^{s}$ not all divisible by $p$; let $1 \leqslant m \leqslant r$ be maximal with $i_{m}$ not divisible by $p$. Then the local sections

$$
\begin{aligned}
& V^{s}\left(\left[x_{1}\right]^{i_{1}} \ldots\left[x_{r}\right]^{i_{r}}[\pi]^{j} d \log x_{m_{1}} \ldots d \log x_{m_{q-1}} d \log \pi\right), \\
& d V^{s}\left(\left[x_{1}\right]^{i_{1}} \ldots\left[x_{r}\right]^{i_{r}}[\pi]^{j} d \log x_{m_{1}^{\prime}} \ldots d \log x_{m_{q-2}^{\prime}} d \log \pi\right),
\end{aligned}
$$

where $1 \leqslant m_{1}<\cdots<m_{q-1} \leqslant r$ and $1 \leqslant m_{1}^{\prime}<\cdots<m_{q-2}^{\prime} \leqslant r$, and where all $m_{i}$ and $m_{i}^{\prime}$ are different from $m$, together with the local sections

$$
[\pi]^{j} d \log x_{m_{1}} \ldots d \log x_{m_{q-1}} d \log \pi,
$$

where $1 \leqslant m_{1}<\cdots<m_{q-1} \leqslant r$, form a basis.

(iii) If $n \leqslant v$ or if $e^{\prime} \leqslant j$, then $\operatorname{Fil}_{U}^{2 j} E_{n}^{q}=0$.

Proof. Let $\Sigma_{n}^{q}$ and $\Gamma_{n}^{q}$ denote the sets of local sections of $E_{n}^{q}$ listed in the statements of Thm. 1.3.5 and of Prop. 1.3.4, respectively. We first construct a bijection $f: \Gamma_{n}^{q} \stackrel{\sim}{\longrightarrow} \Sigma_{n}^{q}$ with the property that $\omega$ and $f(\omega)$ are similar in the sense of Def. 1.3.3. This proves that $\Sigma_{n}^{q}$ is an $\mathcal{O}_{Y}$-module basis of $E_{n}^{q}$.

Let $0 \leqslant s<n$, let $0 \leqslant i_{1}, \ldots, i_{r}<p^{s}$, and let $0 \leqslant j<e$. We let

$$
v=\min \left\{s, v_{p}\left(i_{1}\right), \ldots, v_{p}\left(i_{r}\right), v_{p}\left(j-e^{\prime}\right)\right\}
$$

and define $s^{\prime}=s-v, i_{m}^{\prime}=p^{-v} i_{m}$, and

$$
j^{\prime}=p^{-v} j+e\left(\frac{p^{-v}-1}{p^{-1}-1}\right)=p^{-v}\left(j+p e\left(\frac{p^{v}-1}{p-1}\right)\right)=p^{-v}\left(j-e^{\prime}\right)+e^{\prime} .
$$

Then $0 \leqslant s^{\prime}<n-v, 0 \leqslant i_{m}^{\prime}<p^{s^{\prime}}$, and $j^{\prime}$ is an integer that satisfies

$$
e\left(\frac{p^{-v}-1}{p^{-1}-1}\right) \leqslant j^{\prime}<e\left(\frac{p^{-(v+1)}-1}{p^{-1}-1}\right) .
$$

The number $j^{\prime}$ is an integer since $j^{\prime} \in \mathbb{Z}\left[\frac{1}{p}\right]$ and $v_{p}\left(j^{\prime}\right) \geqslant 0$.

Conversely, let $0 \leqslant j^{\prime}<e^{\prime}$, and let $v$ be the unique integer given by the above pair of inequalities. Also let $0 \leqslant s^{\prime}<n-v$ and $0 \leqslant i_{1}^{\prime}, \ldots, i_{r}^{\prime}<p^{s^{\prime}}$ be given. We define $s=s^{\prime}+v, i_{m}=p^{v} i_{m}^{\prime}$, and

$$
j=p^{v}\left(j^{\prime}-e\left(\frac{p^{-v}-1}{p^{-1}-1}\right)\right)=p^{v} j^{\prime}-p e\left(\frac{p^{v}-1}{p-1}\right)=p^{v}\left(j^{\prime}-e^{\prime}\right)+e^{\prime} .
$$

Then $v \leqslant s<n, 0 \leqslant i_{m}<p^{s}$, and $j$ is an integer and $0 \leqslant j<e$. We define the bijection $f: \Gamma_{n}^{q} \rightarrow \Sigma_{n}^{q}$ by considering several cases. In each case the similarity of $\omega$ and $f(\omega)$ follows by iterated use of the similarity $[\pi]^{e} \doteq V(1)$. 
First, suppose that not all $i_{m}, 1 \leqslant m \leqslant r$, are equal to zero and that $v<v_{p}\left(i_{m}\right)$, for all $1 \leqslant m \leqslant r$. Then $f$ takes the local sections

$$
\begin{aligned}
& V^{s}\left(\left[x_{1}\right]^{i_{1}} \ldots\left[x_{r}\right]^{i_{r}}[\pi]^{j} d \log x_{m_{1}} \ldots d \log x_{m_{q}}\right), \\
& d V^{s}\left(\left[x_{1}\right]^{i_{1}} \ldots\left[x_{r}\right]^{i_{r}}[\pi]^{j} d \log x_{m_{1}} \ldots d \log x_{m_{q-1}}\right),
\end{aligned}
$$

where $1 \leqslant m_{1}<\cdots<m_{q} \leqslant r$ (resp. $1 \leqslant m_{1}<\cdots<m_{q-1} \leqslant r$ ), to the similar local sections

$$
\begin{aligned}
& V^{s^{\prime}}\left(\left[x_{1}\right]^{i_{1}^{\prime}} \ldots\left[x_{r}\right]_{r}^{i_{r}^{\prime}}[\pi]^{j^{\prime}} d \log x_{m_{1}} \ldots d \log x_{m_{q}}\right), \\
& d V^{s^{\prime}}\left(\left[x_{1}\right]^{i_{1}^{\prime}} \ldots\left[x_{r}\right]^{i_{r}^{\prime}}[\pi]^{j^{\prime}} d \log x_{m_{1}} \ldots d \log x_{m_{q-1}}\right) .
\end{aligned}
$$

We note that $0<s^{\prime}<n-v$, that all $i_{m}^{\prime}, 1 \leqslant m \leqslant r$, are divisible by $p$ and that $j^{\prime}$ is not divisible by $p$.

Next, suppose that not all $i_{m}, 1 \leqslant m \leqslant r$, are zero and that $v=v_{p}\left(i_{m}\right)$, for some $1 \leqslant m \leqslant r$. Let $m$ be maximal with this property. Then $f$ takes the local sections

$$
\begin{aligned}
& V^{s}\left(\left[x_{1}\right]^{i_{1}} \ldots\left[x_{r}\right]^{i_{r}}[\pi]^{j} d \log x_{m_{1}} \ldots d \log x_{m_{q}}\right), \\
& V^{s}\left(\left[x_{1}\right]^{i_{1}} \ldots\left[x_{r}\right]^{i_{r}}[\pi]^{j} d \log x_{m_{1}} \ldots d \log x_{m_{q-1}} d \log \pi\right), \\
& d V^{s}\left(\left[x_{1}\right]^{i_{1}} \ldots\left[x_{r}\right]^{i_{r}}[\pi]^{j} d \log x_{m_{1}} \ldots d \log x_{m_{q-1}}\right), \\
& d V^{s}\left(\left[x_{1}\right]^{i_{1}} \ldots\left[x_{r}\right]^{i_{r}}[\pi]^{j} d \log x_{m_{1}} \ldots d \log x_{m_{q-2}} d \log \pi\right),
\end{aligned}
$$

where $1 \leqslant m_{1}<\cdots<m_{q} \leqslant r$ (resp. $1 \leqslant m_{1}<\cdots<m_{q-1} \leqslant r$, resp. $1 \leqslant m_{1}<$ $\left.\cdots<m_{q-2} \leqslant r\right)$, and where all $m_{i} \neq m$, to the similar local sections

$$
\begin{aligned}
& V^{s^{\prime}}\left(\left[x_{1}\right]^{i_{1}^{\prime}} \ldots\left[x_{r}\right]^{i_{r}^{\prime}}[\pi]^{j^{\prime}} d \log x_{m_{1}} \ldots d \log x_{m_{q}}\right), \\
& V^{s^{\prime}}\left(\left[x_{1}\right]^{i_{1}^{\prime}} \ldots\left[x_{r}\right]^{i_{r}^{\prime}}[\pi]^{j^{\prime}} d \log x_{m_{1}} \ldots d \log x_{m_{q-1}} d \log \pi\right), \\
& d V^{s^{\prime}}\left(\left[x_{1}\right]^{i_{1}^{\prime}} \ldots\left[x_{r}\right]^{i_{r}^{\prime}}[\pi]^{j^{\prime}} d \log x_{m_{1}} \ldots d \log x_{m_{q-1}}\right), \\
& d V^{s^{\prime}}\left(\left[x_{1}\right]^{i_{1}^{\prime}} \ldots\left[x_{r}\right]^{i_{r}^{\prime}}[\pi]^{j^{\prime}} d \log x_{m_{1}} \ldots d \log x_{m_{q-2}} d \log \pi\right) .
\end{aligned}
$$

We note that $0<s^{\prime}<n-v$, that $i_{m}^{\prime}$ is not divisible by $p$, and that $j^{\prime}$ may or may not be divisible by $p$.

Next, if all $i_{m}, 1 \leqslant m \leqslant r$, are zero and if $v<s$, then $f$ takes the local sections

$$
\begin{aligned}
& V^{s}\left([\pi]^{j} d \log x_{m_{1}} \ldots d \log x_{m_{q}}\right), \\
& d V^{s}\left([\pi]^{j} d \log x_{m_{1}} \ldots d \log x_{m_{q-1}}\right),
\end{aligned}
$$

where $1 \leqslant m_{1}<\cdots<m_{q} \leqslant r$ (resp. $1 \leqslant m_{1}<\cdots<m_{q-1} \leqslant r$ ) to the similar local sections

$$
\begin{aligned}
& V^{s^{\prime}}\left([\pi]^{j^{\prime}} d \log x_{m_{1}} \ldots d \log x_{m_{q}}\right), \\
& d V^{s^{\prime}}\left([\pi]^{j^{\prime}} d \log x_{m_{1}} \ldots d \log x_{m_{q-1}}\right) .
\end{aligned}
$$

We note that $0<s^{\prime}<n-v$ and that $j^{\prime}$ is not divisible by $p$.

Finally, if all $i_{m}, 1 \leqslant m \leqslant r$, are zero and if $v=s$, then $f$ takes the local sections

$$
\begin{aligned}
& V^{s}\left([\pi]^{j} d \log x_{m_{1}} \ldots d \log x_{m_{q}}\right), \\
& V^{s}\left([\pi]^{j} d \log x_{m_{1}} \ldots d \log x_{m_{q-1}} d \log \pi\right),
\end{aligned}
$$


where $1 \leqslant m_{1}<\cdots<m_{q} \leqslant r$ (resp. $1 \leqslant m_{1}<\cdots<m_{q-1} \leqslant r$ ) to the similar local sections

$$
\begin{aligned}
& {[\pi]^{j^{\prime}} d \log x_{m_{1}} \ldots d \log x_{m_{q}},} \\
& {[\pi]^{j^{\prime}} d \log x_{m_{1}} \ldots d \log x_{m_{q-1}} d \log \pi .}
\end{aligned}
$$

The integer $j^{\prime}$ may or may not be divisible by $p$. This completes the definition of the map $f: \Gamma_{n}^{q} \rightarrow \Sigma_{n}^{q}$. It is clear that $f$ is a bijection.

It remains to show that the $U$-filtration of $E_{n}^{q}$ is as stated. Let $A^{m, q} \subset E_{n}^{q}$ be the sub- $\mathcal{O}_{Y}$-module generated by those elements of $\Sigma_{n}^{q}$ that are listed in the statement of the theorem as having $U$-filtration greater than or equal to $m$. It is then clear that $A^{m, q} \subset U^{m, q}=\operatorname{Fil}_{U}^{m} E_{n}^{q}$ and we must show that also $U^{m, q} \subset A^{m, q}$. We recall that if $m=2 j$ (resp. if $m=2 j+1$ ), then $U^{m, *}$ is the differential graded ideal generated by $i^{*} \bar{W}_{n}\left(\mathfrak{m}^{j} \mathcal{O}_{X}\right) \subset i^{*} \bar{W}_{n}\left(\mathcal{O}_{X}\right)$ (resp. by $i^{*} \bar{W}_{n}\left(\mathfrak{m}^{j} \mathcal{O}_{X}\right) \cdot d \log M_{X} \subset i^{*} \bar{W}_{n} \Omega_{\left(X, M_{X}\right)}^{1}$ and $\left.i^{*} \bar{W}_{n}\left(\mathfrak{m}^{j+1} \mathcal{O}_{X}\right) \subset i^{*} \bar{W}_{n}\left(\mathcal{O}_{X}\right)\right)$. So it suffices to show that $i^{*} \bar{W}_{n}\left(\mathfrak{m}^{j} \mathcal{O}_{X}\right) \subset A^{2 j, 0}$, that the product takes $A^{m, q} \otimes A^{m^{\prime}, q^{\prime}}$ to $A^{m+m^{\prime}, q+q^{\prime}}$, and that the differential takes $A^{m, q}$ to $A^{m, q+1}$. The second statement is verified by explicitly calculating the products of basis elements of $A^{m, q}$ and $A^{m^{\prime}, q^{\prime}}$ in a manner similar to [11, Sect. 4], and the last statement is immediate. We verify the first statement.

One sees as in the proof of Prop. 1.3.4 that $U^{2 j, 0}=i^{*} \bar{W}_{n}\left(\mathfrak{m}^{j} \mathcal{O}_{X}\right)$ is generated as an $\mathcal{O}_{Y}$-module by the local sections

$$
V^{s}\left(\left[x_{1}\right]^{i_{1}} \ldots\left[x_{r}\right]^{i_{r}}[\pi]^{a}\right),
$$

where $0 \leqslant s<n, 0 \leqslant i_{1}, \ldots, i_{r}<p^{s}$, and $j \leqslant a<j+e$. Iterated use of the similarity $[\pi]^{e} \doteq V(1)$ shows that the generators with $e^{\prime} \leqslant a<j+e$ are equal to zero. For the remaining generators we again let

$$
v=\min \left\{s, v_{p}\left(i_{1}\right), \ldots, v_{p}\left(i_{r}\right), v_{p}\left(a-e^{\prime}\right)\right\}
$$

and define $s^{\prime}=s-v, i_{m}^{\prime}=p^{-v} i_{m}$, and $a^{\prime}=p^{-v}\left(a-e^{\prime}\right)+e^{\prime}$. Then

$$
V^{s}\left(\left[x_{1}\right]^{i_{1}} \ldots\left[x_{r}\right]^{i_{r}}[\pi]^{a}\right) \doteq V^{s^{\prime}}\left(\left[x_{1}\right]^{i_{1}^{\prime}} \ldots\left[x_{r}\right]^{i_{r}^{\prime}}[\pi]^{a^{\prime}}\right)
$$

and hence the local sections on the right-hand side generate $U^{2 j, 0}$ as an $\mathcal{O}_{Y^{-}}$-module. Here $0 \leqslant s^{\prime}<n-v, 0 \leqslant i_{1}^{\prime}, \ldots, i_{r}^{\prime}<p^{s^{\prime}}$, and $j \leqslant a^{\prime}<e^{\prime}$, and if $s^{\prime}>0$, then not all of $i_{1}^{\prime}, \ldots, i_{r}^{\prime}$ and $a^{\prime}$ are divisible by $p$. These local sections are all contained in $A^{2 j, 0}$ and hence $U^{2 j, 0} \subset A^{2 j, 0}$ as desired.

Addendum 1.3.6. There is a natural exact sequence

$$
0 \rightarrow i^{*} \bar{W}_{n} \Omega_{X}^{q} \stackrel{j_{*}}{\rightarrow} i^{*} \bar{W}_{n} \Omega_{\left(X, M_{X}\right)}^{q} \stackrel{\partial}{\rightarrow} \bar{W}_{n} \Omega_{Y}^{q-1} \rightarrow 0 .
$$

Proof. The map $j_{*}$ is induced by the canonical map from $X$ with the trivial logstructure to $X$ with the canonical log-structure. To construct the map $\partial$ we first show that the map

$$
f_{\pi}: W_{n} \Omega_{Y}^{q} \oplus W_{n} \Omega_{Y}^{q-1} \rightarrow W_{n} \Omega_{\left(Y, M_{Y}\right)}^{q}
$$

that to $\left(\omega, \omega^{\prime}\right)$ assigns $\omega+\omega^{\prime} d \log _{n} \pi$ is an isomorphism. Since the statement is local for the étale topology, it suffices to consider $Y=\mathbb{A}_{k}^{r}$. This case follows inductively from the trivial case $r=0$ since the domain and target for $Y=\mathbb{A}_{k}^{r}$ are given by the same formula [11, Thm. B] in terms of the domain and target for $Y=\mathbb{A}_{k}^{r-1}$. We now define $\partial$ to be the composite map

$$
i^{*} W_{n} \Omega_{\left(X, M_{X}\right)}^{q} \rightarrow W_{n} \Omega_{\left(Y, M_{Y}\right)}^{q} \stackrel{f_{\pi}}{\sim} W_{n} \Omega_{Y}^{q} \oplus W_{n} \Omega_{Y}^{q-1} \rightarrow W_{n} \Omega_{Y}^{q-1},
$$


where the left-hand map is the canonical projection and where the right-hand map is the projection onto the second summand. We note that $\partial$ is independent of the choice of uniformizer since $f_{\pi}\left(\omega, \omega^{\prime}\right)=f_{u \pi}\left(\omega+\omega^{\prime} d \log _{n} u, \omega^{\prime}\right)$. It also is clear from the definition that the composite $\partial \circ j_{*}$ is equal to zero.

Let $x_{1}, \ldots, x_{r}$ be local coordinates of an open neighborhood on $X$ around a point of $Y$, and let $\bar{x}_{1}, \ldots, \bar{x}_{r}$ be the corresponding local coordinates on $Y$. Lemma 1.3.1 allows us to view the sequence of the statement as a sequence of $\mathcal{O}_{Y}$-modules, and Thm. 1.3.5 gives a basis of the middle term. The map $\partial$ takes

$$
\begin{gathered}
\partial\left(V^{s}\left(\left[x_{1}\right]^{i_{1}} \ldots\left[x_{r}\right]^{i_{r}} d \log x_{m_{1}} \ldots d \log x_{m_{q-1}} d \log \pi\right)\right) \\
\quad=V^{s}\left(\left[\bar{x}_{1}\right]^{i_{1}} \ldots\left[\bar{x}_{r}\right]^{i_{r}} d \log \bar{x}_{m_{1}} \ldots d \log \bar{x}_{m_{q-1}}\right), \\
\partial\left(d V^{s}\left(\left[x_{1}\right]^{i_{1}} \ldots\left[x_{r}\right]^{i_{r}} d \log x_{m_{1}} \ldots d \log {x_{x_{q-2}}} d \log \pi\right)\right) \\
\quad=d V^{s}\left(\left[\bar{x}_{1}\right]^{i_{1}} \ldots\left[\bar{x}_{r}\right]^{i_{r}} d \log \bar{x}_{m_{1}} \ldots d \log \bar{x}_{m_{q-2}}\right),
\end{gathered}
$$

and annihilates all remaining basis elements. One shows, in a manner similar to the proof of Prop. 1.3.2 that, Zariski locally on $Y$, the sheaves $i^{*} \bar{W}_{n} \Omega_{X}^{q}$ and $\bar{W}_{n} \Omega_{Y}^{q-1}$ with the $\mathcal{O}_{Y}$-module structure given by Lemma 1.3.1 are free and that their ranks satisfy the equation

$$
\operatorname{rk}_{\mathcal{O}_{Y}} i^{*} \bar{W}_{n} \Omega_{X}^{q}+\operatorname{rk}_{\mathcal{O}_{Y}} \bar{W}_{n} \Omega_{Y}^{q-1}=\operatorname{rk}_{\mathcal{O}_{Y}} i^{*} \bar{W}_{n} \Omega_{\left(X, M_{X}\right)}^{q} .
$$

This completes the proof.

1.4. We end this section with the following result on the structure of the higher torsion in the de Rham-Witt complex. The proof we give here uses the cyclotomic trace; see [9. It would be desirable to have a purely algebraic proof.

Proposition 1.4.1. If $\mu_{p^{v}} \subset K$, then for all $0 \leqslant m<v$ and all $q \geqslant 0$, multiplication by $p^{m}$ induces an isomorphism of sheaves of pro-abelian groups

$$
\bar{W} \cdot \Omega_{\left(X, M_{X}\right)}^{q}=\operatorname{gr}_{p}^{0} W \cdot \Omega_{\left(X, M_{X}\right)}^{q} \stackrel{\sim}{\longrightarrow} \operatorname{gr}_{p}^{m} W \cdot \Omega_{\left(X, M_{X}\right)}^{q} .
$$

Proof. We must show that for all $0 \leqslant m<v$ and all $q \geqslant 0$, the following sequence of sheaves of pro-abelian groups on the small étale site of $X$ is exact:

$$
0 \rightarrow W \cdot \Omega_{\left(X, M_{X}\right)}^{q} / p \stackrel{p^{m}}{\longrightarrow} W \cdot \Omega_{\left(X, M_{X}\right)}^{q} / p^{m+1} \stackrel{\mathrm{pr}}{\longrightarrow} W \cdot \Omega_{\left(X, M_{X}\right)}^{q} / p^{m} \rightarrow 0 .
$$

This is equivalent to the statement that for all $0 \leqslant m<v$ and all $q, s \geqslant 0$, the following sequence of pro-abelian groups is exact:

$$
0 \rightarrow W \cdot \Omega_{\left(X, M_{X}\right)}^{q-2 s} \otimes \mu_{p}^{\otimes s} \rightarrow W \cdot \Omega_{\left(X, M_{X}\right)}^{q-2 s} \otimes \mu_{p^{m+1}}^{\otimes s} \rightarrow W \cdot \Omega_{\left(X, M_{X}\right)}^{q-2 s} \otimes \mu_{p^{m}}^{\otimes s} \rightarrow 0 .
$$

We need only show that the left-hand map is a monomorphism of pro-abelian groups. To this end we recall that for all $0 \leqslant m<v$ and all $q \geqslant 0$, [11, Thm. E] gives an isomorphism of pro-abelian groups

$$
\bigoplus_{s \geqslant 0} W \cdot \Omega_{\left(X, M_{X}\right)}^{q-2 s} \otimes \mu_{p^{m}}^{\otimes s} \stackrel{\sim}{\longrightarrow} \mathrm{TR}_{q}^{\cdot}\left(X \mid X_{K} ; p, \mathbb{Z} / p^{m}\right) .
$$

In particular, for all $0 \leqslant m<v$ and $q \geqslant 0$, the map induced from the reduction

$$
\operatorname{TR}_{q}^{\cdot}\left(X \mid X_{K} ; p, \mathbb{Z} / p^{m+1}\right) \rightarrow \operatorname{TR}_{q}^{\cdot}\left(X \mid X_{K} ; p, \mathbb{Z} / p^{m}\right)
$$


is an epimorphism of pro-abelian groups. It follows that the long-exact coefficient sequence breaks up into short-exact sequences of pro-abelian groups:

$0 \rightarrow \mathrm{TR}_{q}^{\cdot}\left(X \mid X_{K} ; p, \mathbb{Z} / p\right) \rightarrow \mathrm{TR}_{q}^{\cdot}\left(X \mid X_{K} ; p, \mathbb{Z} / p^{m+1}\right) \rightarrow \mathrm{TR}_{q}^{\cdot}\left(X \mid X_{K} ; p, \mathbb{Z} / p^{m}\right) \rightarrow 0$.

The proposition follows.

\section{2. $p$-ADIC VANISHING CYCLES}

2.1. Let $\left(i^{*} \bar{W}_{n} \Omega_{\left(X, M_{X}\right)}^{q}\right)^{F=1}$ and $\left(i^{*} \bar{W}_{n} \Omega_{\left(X, M_{X}\right)}^{q}\right)_{F=1}$ denote the kernel and cokernel, respectively, of the map

$$
R-F: i^{*} \bar{W}_{n} \Omega_{\left(X, M_{X}\right)}^{q} \rightarrow i^{*} \bar{W}_{n-1} \Omega_{\left(X, M_{X}\right)}^{q}
$$

of sheaves of abelian groups on the small étale site of $Y$. We consider these sheaves both in the Nisnevich topology and the étale topology. The $U$-filtration is preserved by $R-F$ and hence induces filtrations of the kernel and cokernel sheaves. We begin with the following observation.

Lemma 2.1.1. Suppose that $m \geqslant 2$. Then, for all integers $n$ and $q$, the map

$$
R-F: \operatorname{Fil}_{U}^{m} \bar{W}_{n} \Omega_{\left(X, M_{X}\right)}^{q} \rightarrow \operatorname{Fil}_{U}^{m} \bar{W}_{n-1} \Omega_{\left(X, M_{X}\right)}^{q}
$$

is a surjective map of pre-sheaves of abelian groups on the small étale site of $Y$.

Proof. We consider the case $m=2 j$; the case $m=2 j+1$ is similar. It suffices to show that if $a_{0}, \ldots, a_{q}$ and $a_{0}^{\prime}, \ldots, a_{q-1}^{\prime}$ are local sections of $\mathcal{O}_{X}$ such that $\operatorname{ord}_{Y}\left(a_{i}\right) \geqslant j$ and $\operatorname{ord}_{Y}\left(a_{i^{\prime}}^{\prime}\right) \geqslant j$, for some $0 \leqslant i \leqslant q$ and $0 \leqslant i^{\prime} \leqslant q-1$, then the following local sections are in the image of $R-F$ :

$$
\begin{aligned}
& V^{s_{0}}\left[a_{0}\right]_{n-1} d V^{s_{1}}\left[a_{1}\right]_{n-1} \ldots d V^{s_{q}}\left[a_{q}\right]_{n-1}, \\
& V^{s_{0}}\left[a_{0}\right]_{n-1} d V^{s_{1}}\left[a_{1}\right]_{n-1} \ldots d V^{s_{q-1}}\left[a_{q-1}\right]_{n-1} d \log _{n-1} \pi .
\end{aligned}
$$

Indeed, every local section of $\operatorname{Fil}_{U}^{m} \bar{W}_{n-1} \Omega_{\left(X, M_{X}\right)}^{q}$ is a sum of such elements. We now use that since $j \geqslant 1$, the following series converge:

$$
\begin{aligned}
& \sum_{t \geqslant 0} F^{t}\left(V^{s_{0}}\left[a_{0}\right]_{n+t} d V^{s_{1}}\left[a_{1}\right]_{n+t} \ldots d V^{s_{q}}\left[a_{q}\right]_{n+t}\right), \\
& \sum_{t \geqslant 0} F^{t}\left(V^{s_{0}}\left[a_{0}\right]_{n+t} d V^{s_{1}}\left[a_{1}\right]_{n+t} \ldots d V^{s_{q-1}}\left[a_{q-1}\right]_{n+t} d \log _{n+t} \pi\right) .
\end{aligned}
$$

The images by $R-F$ of the sums of these series are equal to the given elements.

Let $\Omega_{Y, \log }^{q} \subset \Omega_{Y}^{q}$ be the subsheaf generated locally for the étale topology on $Y$ by the local sections of the form $d \log y_{1} \ldots d \log y_{q}$. If $y$ is a local section of $\mathcal{O}_{Y}$, we denote by $\tilde{y}$ any lifting of $y$ to a local section of $i^{*} \mathcal{O}_{X}$.

Theorem 2.1.2. The sheaf $M^{q}=\left(i^{*} \bar{W} \cdot \Omega_{\left(X, M_{X}\right)}^{q}\right)^{F=1}$ of pro-abelian groups on the small étale site of $Y$ has the following structure.

(i) There is an isomorphism

$$
\left.\rho_{0}: \Omega_{Y, \log }^{q} \stackrel{\sim}{\longrightarrow} \operatorname{gr}_{U}^{0} M^{q} \quad \text { (resp. } \rho_{1}: \Omega_{Y, \log }^{q-1} \stackrel{\sim}{\longrightarrow} \operatorname{gr}_{U}^{1} M^{q}{ }^{q}\right)
$$

that to $d \log y_{1} \ldots d \log y_{q} \quad\left(\right.$ resp. $\left.d \log y_{1} \ldots d \log y_{q-1}\right)$ assigns $d \log \tilde{y}_{1} \ldots d \log \tilde{y}_{q}$ (resp. $d \log \tilde{y}_{1} \ldots d \log \tilde{y}_{q-1} d \log \pi$ ).

(ii) If $0<j<e^{\prime}$, and if $p$ does not divide $j$ (resp. if $p$ divides $j$ ), there is an isomorphism

$$
\rho_{2 j}: \Omega_{Y}^{q-1} / B \Omega_{Y}^{q-1} \stackrel{\sim}{\longrightarrow} \operatorname{gr}_{U}^{2 j} M_{\cdot}^{q} \quad\left(\text { resp. } \rho_{2 j}: \Omega_{Y}^{q-1} / Z \Omega_{Y}^{q-1} \stackrel{\sim}{\longrightarrow} \operatorname{gr}_{U}^{2 j} M_{.}^{q}\right)
$$


that to ad $\log y_{1} \ldots d \log y_{q-1}$ assigns $d \log \left(1+\pi^{j} \tilde{a}\right) d \log \tilde{y}_{1} \ldots d \log \tilde{y}_{q-1}$.

(iii) If $0<j<e^{\prime}$, there is an isomorphism

$$
\rho_{2 j+1}: \Omega_{Y}^{q-2} / Z \Omega_{Y}^{q-2} \stackrel{\sim}{\longrightarrow} \operatorname{gr}_{U}^{2 j+1} M^{q}
$$

that takes ad $\log y_{1} \ldots d \log y_{q-2}$ to $d \log \left(1+\pi^{j} \tilde{a}\right) d \log \tilde{y}_{1} \ldots d \log \tilde{y}_{q-2} d \log \pi$.

(iv) If $e^{\prime} \leqslant j$, then $\mathrm{Fil}_{U}^{2 j} M_{.}^{q}$ is equal to zero.

Proof. It follows from Prop. B.1.1 and from [3, Sect. 4] that the maps $\rho_{m}$ of the statement are well-defined strict maps of sheaves of pro-abelian groups. We first consider the statement (i). We abbreviate $E_{n}^{q}=i^{*} \bar{W}_{n} \Omega_{\left(X, M_{X}\right)}^{q}$ as before. It follows from Lemma 2.1.1 that there is an exact sequence of sheaves of abelian groups

$$
0 \rightarrow M_{n}^{q} / \mathrm{Fil}_{U}^{2} M_{n}^{q} \rightarrow E_{n}^{q} / \mathrm{Fil}_{U}^{2} E_{n}^{q} \stackrel{R-F}{\longrightarrow} E_{n-1}^{q} / \mathrm{Fil}_{U}^{2} E_{n-1}^{q} .
$$

Moreover, Lemma 1.2.1 identifies the middle and right-hand terms with the reduction modulo $p$ of the de Rham-Witt complex of $\left(Y, M_{Y}\right)$. Hence, we have an isomorphism of sheaves of pro-abelian groups

$$
M_{\cdot}^{q} / \mathrm{Fil}_{U}^{2} M_{\cdot}^{q} \stackrel{\sim}{\longrightarrow}\left(\bar{W} \cdot \Omega_{\left(Y, M_{Y}\right)}^{q}\right)^{F=1} .
$$

The structure of the right-hand side is well-understood; see [24, Prop. 2.4.1]. The statement for $\operatorname{gr}_{U}^{0} M^{q}$. and $\operatorname{gr}_{U}^{1} M^{q}$. follows.

We next prove the statement (ii). It follows again from Lemma 2.1.1 that there is a short-exact sequence of sheaves of abelian groups

$$
0 \rightarrow \operatorname{gr}_{U}^{2 j} M_{n}^{q} \rightarrow \operatorname{gr}_{U}^{2 j} E_{n}^{q} \stackrel{R-F}{\longrightarrow} \operatorname{gr}_{U}^{2 j} E_{n-1}^{q} \rightarrow 0 .
$$

We recall that if $x_{1}, \ldots, x_{r}$ are local coordinates of an open neighborhood on $X$ around a point of $Y$, then, in the corresponding open neighborhood of $Y$, the sheaf $\operatorname{gr}_{U}^{2 j} E_{n}^{q}$ has the structure of a free $\mathcal{O}_{Y}$-module with a basis given by the local sections

$$
\begin{aligned}
& V^{s}\left(\left[x_{1}\right]^{i_{1}} \ldots\left[x_{r}\right]^{i_{r}}[\pi]^{j} d \log x_{m_{1}} \ldots d \log x_{m_{q}}\right), \\
& d V^{s}\left(\left[x_{1}\right]^{i_{1}} \ldots\left[x_{r}\right]^{i_{r}}[\pi]^{j} d \log x_{m_{1}} \ldots d \log x_{m_{q-1}}\right),
\end{aligned}
$$

where $0 \leqslant s<n-v$ and $0<s<n-v$, respectively, and where the multi-indices $i$ and $m$ vary as in the statement of Thm. 1.3.5 (ii). We consider the short-exact sequence of $\mathcal{O}_{Y}$-modules

$$
0 \rightarrow\left(\operatorname{gr}_{U}^{2 j} E_{n}^{q}\right)^{\prime} \rightarrow \operatorname{gr}_{U}^{2 j} E_{n}^{q} \rightarrow\left(\operatorname{gr}_{U}^{2 j} E_{n}^{q}\right)^{\prime \prime} \rightarrow 0
$$

where the left-hand term is defined to be the sub- $\mathcal{O}_{Y}$-module spanned by the basis elements in the top line above. The images of the basis elements in the bottom line above form an $\mathcal{O}_{Y}$-basis of the right-hand term. Moreover, the map $R-F$ gives rise to a map of short-exact sequences of sheaves of abelian groups as follows:

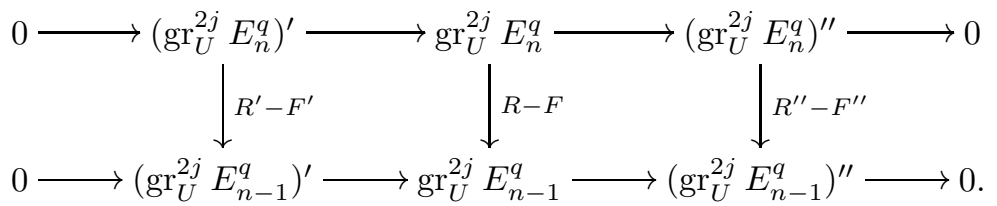

The map $R^{\prime}$ is $\mathcal{O}_{Y}$-linear, annihilates the basis elements with $s=n-v-1$, and leaves the remaining basis elements unchanged, and the map $F^{\prime}$ is equal to zero. It 
follows that $R^{\prime}-F^{\prime}=R^{\prime}$ is surjective, and hence we have the following short-exact sequence of kernels of the vertical maps in the diagram above:

$$
0 \rightarrow\left(\operatorname{gr}_{U}^{2 j} M_{n}^{q}\right)^{\prime} \rightarrow \operatorname{gr}_{U}^{2 j} M_{n}^{q} \rightarrow\left(\operatorname{gr}_{U}^{2 j} M_{n}^{q}\right)^{\prime \prime} \rightarrow 0 .
$$

Moreover, as $n$ varies, the left-hand term is zero as a sheaf of pro-abelian groups. Indeed, the structure maps are zero. Similarly, the map $R^{\prime \prime}$ above is the $\mathcal{O}_{Y}$-linear map that annihilates the basis elements with $s=n-v-1$ and leaves the remaining basis elements unchanged. The map $F^{\prime \prime}$ is the $\varphi$-linear map that annihilates the basis elements with $s=1$ and that is given on the remaining basis elements by

$$
\begin{aligned}
& F\left(d V^{s}\left(\left[x_{1}\right]^{i_{1}} \ldots\left[x_{r}\right]^{i_{r}}[\pi]^{j} d \log x_{m_{1}} \ldots d \log x_{m_{q-1}}\right)\right) \\
& =\bar{x}_{1}^{k_{1}} \ldots \bar{x}_{r}^{k_{r}} d V^{s-1}\left(\left[x_{1}\right]^{i_{1}^{\prime}} \ldots\left[x_{r}\right]^{i_{r}^{\prime}}[\pi]^{j} d \log x_{m_{1}} \ldots d \log x_{m_{q-1}}\right),
\end{aligned}
$$

where $i_{m}=k_{m} p^{s-1}+i_{m}^{\prime}$ with $0 \leqslant i_{1}^{\prime}, \ldots, i_{r}^{\prime}<p^{s-1}$. It follows that a local section

$$
\omega=\sum_{s, m, i} a_{m, i}^{(s)} d V^{s}\left([x]_{1}^{i_{1}} \ldots\left[x_{r}\right]^{i_{r}}[\pi]^{j} d \log x_{m_{1}} \ldots d \log x_{m_{q-1}}\right)
$$

of $\operatorname{gr}_{U}^{2 j} E_{n}^{\prime \prime q}$ lies in $\operatorname{gr}_{U}^{2 j} M_{n}^{\prime \prime q}$ if and only if the local sections $a_{m, i}^{(s)}$ of $\mathcal{O}_{Y}$ satisfy the following system of equations:

$$
a_{m, i}^{(s-1)}=\sum_{0 \leqslant k_{1}, \ldots, k_{r}<p}\left(a_{m, k p^{s-1}+i}^{(s)}\right)^{p} \bar{x}_{1}^{k_{1}} \ldots \bar{x}_{r}^{k_{r}}
$$

Here $1<s<n-v$ and the multi-indices $m$ and $i$ vary as in the statement of Thm.1.3.5 (ii). We note that a solution to this system of equations determines and is determined by the local sections defined by the formula

$$
a_{m}=\sum_{i}\left(a_{m, i}^{(s)}\right)^{p^{s}} \bar{x}_{1}^{i_{1}} \ldots \bar{x}_{r}^{i_{r}} .
$$

In this sum, the multi-index $i$ ranges as in the statement of Thm.1.3.5(ii) depending on the multi-index $m$, which is fixed, and the index $1 \leqslant s<n-v$, which is arbitrary but fixed. It follows that the restriction map

$$
R^{\prime \prime}:\left(\operatorname{gr}_{U}^{2 j} M_{n+1}^{q}\right)^{\prime \prime} \rightarrow\left(\operatorname{gr}_{U}^{2 j} M_{n}^{q}\right)^{\prime \prime}
$$

is an isomorphism, if $n>v+1$, and that $\left(\operatorname{gr}_{U}^{2 j} M_{n}^{q}\right)^{\prime \prime}$ is zero, if $n \leqslant v+1$. Hence, the statement (ii) is equivalent to the statement that, for $n>v+1$, the maps

$$
\bar{\rho}_{2 j}: \Omega_{Y}^{q-1} / B \Omega_{Y}^{q-1} \rightarrow\left(\operatorname{gr}_{U}^{2 j} M_{n}^{q}\right)^{\prime \prime} \quad\left(\text { resp. } \bar{\rho}_{2 j}: \Omega_{Y}^{q-1} / Z \Omega_{Y}^{q-1} \rightarrow\left(\operatorname{gr}_{U}^{2 j} M_{n}^{q}\right)^{\prime \prime}\right)
$$

induced by $\rho_{2 j}$ are isomorphisms of sheaves of abelian groups. To prove this, we note that, for every positive integer $s, \Omega_{Y}^{q-1} / B \Omega_{Y}^{q-1}$ (resp. $\Omega_{Y}^{q-1} / Z \Omega_{Y}^{q-1}$ ) has a canonical structure of a locally free $\mathcal{O}_{Y}^{p^{s}}$-module. If $x_{1}, \ldots, x_{r}$ are the local coordinates of a neighborhood of $X$ that we considered above, then, in the corresponding neighborhood of $Y$, an $\mathcal{O}_{Y}^{p^{s}}$-basis is given by the local sections

$$
\bar{x}_{1}^{i_{1}} \ldots \bar{x}_{r}^{i_{r}} d \log \bar{x}_{m_{1}} \ldots d \log \bar{x}_{m_{q-1}}
$$

where $0 \leqslant i_{1}, \ldots, i_{r}<p^{s}$ (resp. $0 \leqslant i_{1}, \ldots, i_{r}<p^{s}$, not all divisible by $p$ ), and where $1 \leqslant m_{1}<\cdots<m_{q-1} \leqslant r$ are such that, if $m$ is largest with $i_{m}$ prime to $p$, then $m_{i} \neq m$, for all $1 \leqslant i \leqslant q-1$. We note that, for $1 \leqslant s<n-v$ fixed, the multi-indices $i$ and $m$ vary in the same way as in the statement of Thm. 1.3.5 (ii). 
Let $\omega$ be a local section of the sheaf $\Omega_{Y}^{q-1} / B \Omega_{Y}^{q-1}$ (resp. $\Omega_{Y}^{q-1} / Z \Omega_{Y}^{q-1}$ ). Then, for every positive integer $s$, we can write $\omega$ as a linear combination

$$
\omega=\sum_{m, i}\left(a_{m, i}^{(s)}\right)^{p^{s}} \bar{x}_{1}^{i_{1}} \ldots \bar{x}_{r}^{i_{r}} d \log \bar{x}_{m_{1}} \ldots d \log \bar{x}_{m_{q-1}}
$$

with respect to this basis. Then the coefficients $a_{m, i}^{(s)}$ are local sections of $\mathcal{O}_{Y}$ and, as the index $1 \leqslant s<n-v$ varies, constitute a solution to the system of equations that define the subsheaf $\operatorname{gr}_{U}^{2 j} M_{n}^{\prime \prime q} \subset \operatorname{gr}_{U}^{2 j} E_{n}^{q}$. Moreover, Lemma 1.2.3 shows that

$$
\bar{\rho}_{2 j}(\omega)=\sum_{s, m, i} a_{m, i}^{(s)} d V^{s}\left(\left[x_{1}\right]^{i_{1}} \ldots\left[x_{r}\right]^{i_{r}}[\pi]^{j} d \log x_{m_{1}} \ldots d \log x_{m_{q-1}}\right),
$$

and hence $\bar{\rho}_{2 j}$ is an isomorphism as stated. The proof of the statement (iii) is completely analogous, and statement (iv) follows from Thm. 1.3.5 (iv).

Addendum 2.1.3. The canonical projection

$$
i^{*}\left(\bar{W} \cdot \Omega_{\left(X, M_{X}\right)}^{q}\right)_{F=1} \rightarrow\left(\bar{W} \cdot \Omega_{\left(Y, M_{Y}\right)}^{q}\right)_{F=1}
$$

is an isomorphism of pre-sheaves of pro-abelian groups on the small étale site of $Y$. Moreover, the associated sheaf for the étale topology is zero.

Proof. We recall from Lemma 2.1.1 that the map

$$
\mathrm{Fil}_{U}^{2} i^{*} \bar{W} \cdot \Omega_{\left(X, M_{X}\right)}^{q} \stackrel{1-F}{\longrightarrow} \operatorname{Fil}_{U}^{2} i^{*} \bar{W} \cdot \Omega_{\left(X, M_{X}\right)}^{q}
$$

is surjective. The isomorphism of the statement now follows from Lemma 1.2.1. Finally, by the proof of Addendum 1.3.6, there is a split-exact sequence

$$
0 \rightarrow \bar{W} \cdot \Omega_{Y}^{q} \rightarrow \bar{W} \cdot \Omega_{\left(Y, M_{Y}\right)}^{q} \rightarrow \bar{W} \cdot \Omega_{Y}^{q-1} \rightarrow 0
$$

and we have from [13, Prop. I.3.26] that, for the étale topology, the map $1-F$ induces surjections of the left and right-hand terms.

Theorem 2.1.4. There is a natural exact sequence

$$
0 \rightarrow i^{*} R^{q} j_{*} \mu_{p}^{\otimes q} \rightarrow i^{*} \bar{W} \cdot \Omega_{\left(X, M_{X}\right)}^{q} \stackrel{1-F}{\longrightarrow} i^{*} \bar{W} \cdot \Omega_{\left(X, M_{X}\right)}^{q} \rightarrow 0
$$

of sheaves of pro-abelian groups on the small étale site of $Y$ in the étale topology.

Proof. We follow Bloch and Kato [3] and construct the left-hand map of the statement by means of the symbol maps

$$
i^{*} R^{q} j_{*} \mu_{p}^{\otimes q} \leftarrow i^{*}\left(M_{X}^{\mathrm{gp}}\right)^{\otimes q} \rightarrow i^{*}\left(\bar{W} \cdot \Omega_{(X, M)}^{q}\right)^{F=1} .
$$

The right-hand map takes a local section $a_{1} \otimes \cdots \otimes a_{q}$ to $d \log a_{1} \ldots d \log a_{q}$ and the left-hand map takes the same local section to the symbol $\left\{a_{1}, \ldots, a_{q}\right\}$. We recall the definition of the latter. By Hilbert's Theorem 90, the Kummer sequence

$$
0 \rightarrow \mu_{p} \rightarrow \mathcal{O}_{U}^{*} \stackrel{p}{\rightarrow} \mathcal{O}_{U}^{*} \rightarrow 0
$$

gives rise to an exact sequence

$$
0 \rightarrow i^{*} j_{*} \mu_{p} \rightarrow i^{*} j_{*} \mathcal{O}_{U}^{*} \stackrel{p}{\rightarrow} i^{*} j_{*} \mathcal{O}_{U}^{*} \stackrel{\partial}{\rightarrow} i^{*} R^{1} j_{*} \mu_{p} \rightarrow 0
$$

of sheaves of abelian groups on the small étale site of $Y$ in the étale topology. The symbol $\{a\}$ is defined as the image of the local section $a$ by the composite

$$
i^{*} M_{X}^{\mathrm{gp}} \stackrel{\sim}{\longrightarrow} i^{*} j_{*} \mathcal{O}_{U}^{*} \stackrel{\partial}{\rightarrow} i^{*} R^{1} j_{*} \mu_{p}
$$

and $\left\{a_{1}, \ldots, a_{q}\right\}$ as the product of $\left\{a_{1}\right\}, \ldots,\left\{a_{q}\right\}$. 
We may assume that the scheme $X$ is connected. Let $\mathcal{V}^{\prime}$ be the strictly henselian local ring of $X$ at the generic point of $Y$, and let $\mathcal{K}^{\prime}$ be the quotient field of $\mathcal{V}^{\prime}$. We let $\tau$ : Spec $\kappa \rightarrow Y$ be the inclusion of the generic point and consider the maps induced by the symbol maps

$$
\tau^{*} i^{*} R^{q} j_{*} \mu_{p}^{\otimes q} \leftarrow \tau^{*} i^{*}\left(M_{X}^{\mathrm{gp}}\right)^{\otimes q} / p \rightarrow \tau^{*}\left(i^{*} \bar{W} \cdot \Omega_{\left(X, M_{X}\right)}^{q}\right)^{F=1} .
$$

The left and right-hand terms are canonically isomorphic to the skyscraper sheafs associated with the pro-abelian groups $\bar{K}_{q}^{M}\left(\mathcal{K}^{\prime}\right)$ and $\left(\bar{W} \cdot \Omega_{\left(\mathcal{V}^{\prime}, M_{\mathcal{V}^{\prime}}\right)}^{q}\right)^{F=1}$, respectively. It follows that the left-hand map is a surjection whose kernel is equal to the subsheaf generated by the sections $a_{1} \otimes \cdots \otimes a_{q}$ with some $a_{i}+a_{j}=1$. By Prop. B.1.1, these sections are annihilated by the right-hand symbol map, so we have an induced map

$$
\tau^{*} i^{*} R^{q} j_{*} \mu_{p}^{\otimes q} \rightarrow \tau^{*}\left(i^{*} \bar{W} \cdot \Omega_{\left(X, M_{X}\right)}^{q}\right)^{F=1} .
$$

This map preserves $U$-filtrations, and [3, Cor. 1.4.1] and Thm. 2.1.2 show that the induced map of filtration quotients is an isomorphism. It follows that the map is an isomorphism.

We consider the following commutative diagram:

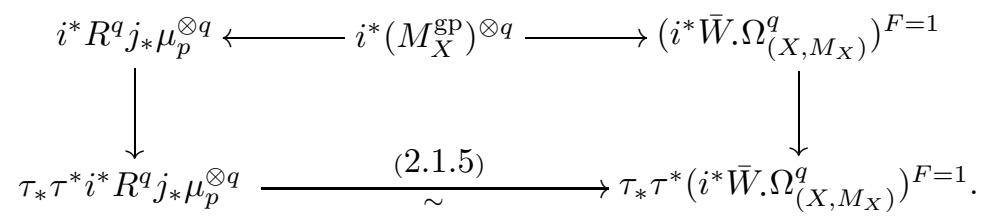

It is proved in [3, Prop. 6.1(i)] that the left-hand vertical map is injective and in op. cit., Cor. 6.1.1, that the upper left-hand horizontal map is surjective. Moreover, the right-hand vertical map is injective, since, Zariski locally on $Y$, the sheaf $i^{*} \bar{W} \cdot \Omega_{\left(X, M_{X}\right)}^{q}$ is a quasi-coherent $\mathcal{O}_{Y}$-module. It follows that the upper horizontal maps have the same kernel, and hence the symbol maps give rise to a map

$$
i^{*} R^{q} j_{*} \mu_{p}^{\otimes q} \rightarrow\left(i^{*} \bar{W} \cdot \Omega_{\left(X, M_{X}\right)}^{q}\right)^{F=1} .
$$

Again this map preserves $U$-filtrations, and [3, Cor. 1.4.1] (see also [24, Prop. 2.4.1]) and Thm. 2.1.2 show that the induced map of the associated graded sheaves is an isomorphism. It follows that the map is an isomorphism as stated.

Remark 2.1.6. It is possible from the proof of Thm. 2.1.2 to derive the following more precise statement about the injectivity of the map

$$
i^{*} R^{q} j_{*} \mu_{p}^{\otimes q} \rightarrow i^{*} \bar{W}_{n} \Omega_{\left(X, M_{X}\right)}^{q} .
$$

As in the statement of Thm. 1.3.5, let $v=v(j)$ be the unique integer such that

$$
e\left(\frac{p^{-v}-1}{p^{-1}-1}\right) \leqslant j<e\left(\frac{p^{-(v+1)}-1}{p^{-1}-1}\right) .
$$

Then the map is injective if $n-1>v$ for all $0 \leqslant j<e^{\prime}$. This, in turn, holds if and only if $p^{n-1}>e^{\prime}$.

Proof of Thm. A. The surjectivity of $1-F$ is an immediate consequence of Addendum 2.1.3. We show by induction on $v \geqslant 1$ that the symbol maps

$$
i^{*} R^{q} j_{*} \mu_{p^{v}}^{\otimes q} \leftarrow i^{*}\left(M_{X}^{\mathrm{gp}}\right)^{\otimes q} / p^{v} \rightarrow\left(i^{*} W \cdot \Omega_{\left(X, M_{X}\right)}^{q} / p^{v}\right)^{F=1}
$$


are surjective and have the same kernel. The case $v=1$ is Thm. 2.1.4. In the induction step we consider the following diagram with exact rows, where we have abbreviated $E^{q}=i^{*} W \cdot \Omega_{\left(X, M_{X}\right)}^{q}$ :

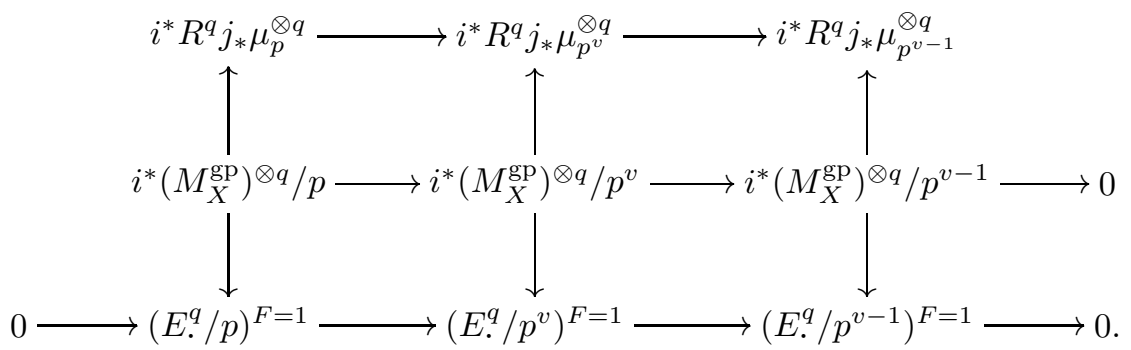

The exactness of the lower row follows from Prop. 1.4.1 and Addendum 2.1.3. By induction the right-hand vertical maps are surjective and have the same kernel. The same is true for the left-hand vertical maps. It follows that the middle vertical maps and the upper right-hand horizontal map are surjective. We claim that the upper left-hand horizontal map is injective. Indeed, this is equivalent, by the long-exact cohomology sequence, to the statement that in the sequence

$$
i^{*} R^{q-1} j_{*} \mu_{p}^{\otimes q} \rightarrow i^{*} R^{q-1} j_{*} \mu_{p^{v}}^{\otimes q} \rightarrow i^{*} R^{q-1} j_{*} \mu_{p^{v-1}}^{\otimes q}
$$

the right-hand map is surjective. But the cup product by a primitive $p^{v}$ th root of unity defines an isomorphism of the sheaves $\mu_{p^{v}}^{\otimes(q-1)}$ and $\mu_{p^{v}}^{\otimes q}$, and we have already proved that the following sequence is exact:

$$
i^{*} R^{q-1} j_{*} \mu_{p}^{\otimes(q-1)} \rightarrow i^{*} R^{q-1} j_{*} \mu_{p^{v}}^{\otimes(q-1)} \rightarrow i^{*} R^{q-1} j_{*} \mu_{p^{v-1}}^{\otimes(q-1)} \rightarrow 0 .
$$

It remains to show that the middle vertical maps in the diagram above have the same kernel. To this end, we assume, as in the proof of Thm. 2.1.4, that $X$ is connected and let $\tau$ : Spec $k \rightarrow Y$ be the inclusion of the generic point. We consider the symbol maps

$$
\tau^{*} i^{*} R^{q} j_{*} \mu_{p^{v}}^{\otimes q} \leftarrow \tau^{*} i^{*}\left(M_{X}^{\mathrm{gp}}\right)^{\otimes q} / p^{v} \rightarrow \tau^{*}\left(i^{*} W \cdot \Omega_{\left(X, M_{X}\right)}^{q} / p^{v}\right)^{F=1} .
$$

The kernel of the left-hand map is generated by the symbols $\left\{a_{1}, \ldots, a_{q}\right\}$ with some $a_{i}+a_{j}=1$, and Prop. B.1.1 shows that these sections are contained in the kernel of the right-hand map. Hence, we have an induced map

$$
\tau^{*} i^{*} R^{q} j_{*} \mu_{p^{v}}^{\otimes q} \rightarrow \tau^{*}\left(i^{*} W \cdot \Omega_{\left(X, M_{X}\right)}^{q} / p^{v}\right)^{F=1} .
$$

It is an isomorphism by induction and by the fact that the upper and lower horizontal rows in the diagram above are short-exact. We consider the following diagram with exact rows:

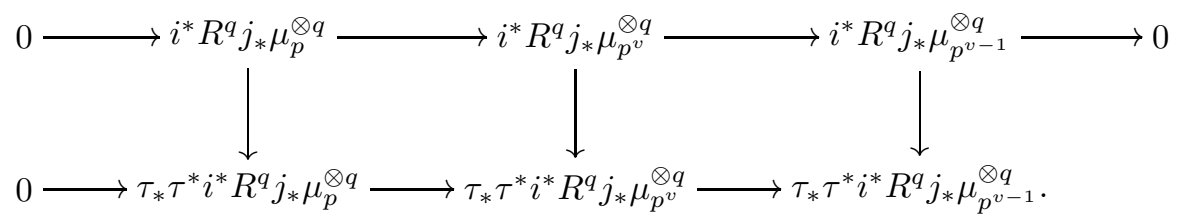

By induction and by [3, Prop. 6.1(i)], the right and left-hand vertical maps are injective. Hence, also the middle vertical map is injective. A similar argument 
shows that also the right-hand vertical map in the following diagram is injective:

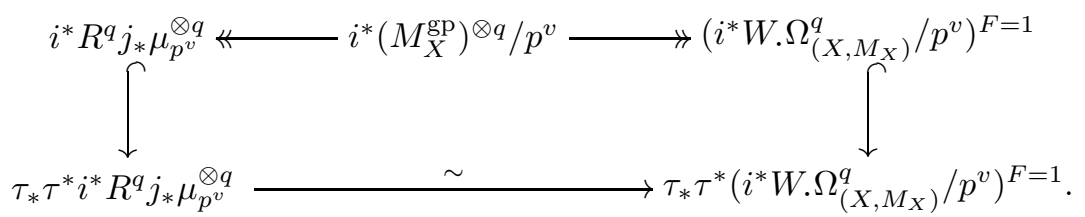

It follows that the upper horizontal maps have the same kernel as desired. This way we obtain the left-hand map of the statement of Thm. A.

$$
i^{*} R^{q} j_{*} \mu_{p^{v}}^{\otimes q} \rightarrow\left(i^{*} W \cdot \Omega_{\left(X, M_{X}\right)}^{q} / p^{v}\right)^{F=1} .
$$

Finally, an induction argument based on the short-exactness of the upper and lower horizontal sequences in the diagram at the beginning of the proof shows that this map is an isomorphism.

\section{Henselian discrete valuation Rings}

3.1. In this section we prove Thm. B of the introduction. The proof uses the following commutative diagram of pro-abelian groups in which the right-hand vertical map is the cyclotomic trace of [4]. We refer the reader to [9] for an introduction and a comprehensive list of references to this construction:

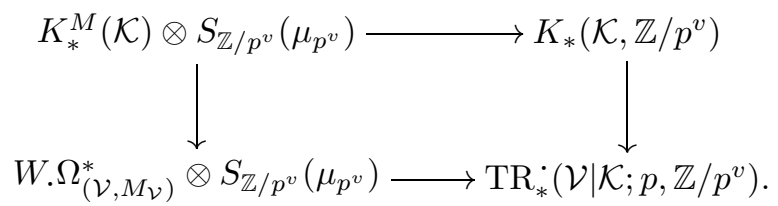

We recall from [10, Thm. C] and [11, Thm. E] that in this diagram the lower horizontal map is an isomorphism of pro-abelian groups.

Suppose first that the residue field $\kappa$ is separably closed. Then Thm. A shows that the left-hand vertical map is injective and an isomorphism onto the Frobenius fixed set of the target. Similarly, we show in Prop. 3.2 .3 below that the right-hand vertical map is injective and an isomorphism onto the Frobenius fixed set of the target. This proves Thm. B in this case.

In the general case the vertical maps in (3.1.1) are not injective, but they still induce surjections onto the Frobenius fixed sets of the respective targets. Hence, to prove Thm. B, we must show that the upper horizontal map induces an isomorphism of the kernel of the left-hand vertical map onto the kernel of the right-hand vertical map. We first express the two kernels in terms of de Rham-Witt groups and then show that the map in question is an isomorphism. The proof of the latter occupies most of the section.

3.2. Let the field $\mathcal{K}$ be as in the statement of Thm. B. We first consider the left-hand vertical map in (3.1.1).

Proposition 3.2.1. Suppose that $\mu_{p} \subset K$. Then there is a natural exact sequence of pro-abelian groups

$$
0 \rightarrow\left(\bar{W} \cdot \Omega_{\left(\mathcal{V}, M_{\mathcal{V}}\right)}^{q-1} \otimes \mu_{p}\right)_{F=1} \rightarrow \bar{K}_{q}^{M}(\mathcal{K}) \rightarrow\left(\bar{W} \cdot \Omega_{\left(\mathcal{V}, M_{\mathcal{V}}\right)}^{q}\right)^{F=1} \rightarrow 0,
$$

where the left-hand map takes the class of $[a] d \log x_{1} \ldots d \log x_{q-1} \otimes \zeta$ to the class of the symbol $\left\{1+a(1-\zeta)^{p}, x_{1}, \ldots, x_{q-1}\right\}$. 
Proof. It follows from [15, Thm. 2(1)] and from Thm.2.1.2 above that the map that to $\left\{a_{1}, \ldots, a_{q}\right\}$ associates $d \log a_{1} \ldots d \log a_{q}$ induces an isomorphism of pro-abelian groups

$$
\bar{K}_{q}^{M}(\mathcal{K}) / \operatorname{Fil}_{U}^{2 e^{\prime}} \bar{K}_{q}(\mathcal{K}) \stackrel{\sim}{\longrightarrow}\left(\bar{W} \cdot \Omega_{\left(\mathcal{V}, M_{\mathcal{V}}\right)}^{q}\right)^{F=1} .
$$

Indeed, the right-hand side is the stalk at the generic point of $Y$ of the sheaf of pro-abelian groups $\left(i^{*} \bar{W} \cdot \Omega_{\left(X, M_{X}\right)}^{q}\right)^{F=1}$ on the small étale site of $Y$ in the Nisnevich topology. Similarly, 15. Thm. 2(1)] and Addendum 2.1.3 shows that the left-hand map of the statement induces an isomorphism of pro-abelian groups

$$
\left(\bar{W} \cdot \Omega_{\left(\mathcal{V}, M_{\mathcal{V}}\right)}^{q-1} \otimes \mu_{p}\right)_{F=1} \stackrel{\sim}{\longrightarrow} \operatorname{Fil}_{U}^{2 e^{\prime}} \bar{K}_{q}^{M}(\mathcal{K}) .
$$

This completes the proof.

Remark 3.2.2. Suppose that $\mu_{p^{v}} \subset K$. One can deduce from Prop. 3.2.1 that there exists a natural exact sequence of pro-abelian groups

$$
0 \rightarrow\left(W \cdot \Omega_{\left(\mathcal{V}, M_{\mathcal{V}}\right)}^{q-1} \otimes \mu_{p^{v}}\right)_{F=1} \rightarrow K_{q}^{M}(\mathcal{K}) / p^{v} \rightarrow\left(W . \Omega_{\left(\mathcal{V}, M_{\mathcal{V}}\right)}^{q} / p^{v}\right)^{F=1} \rightarrow 0 .
$$

However, we do not have a purely algebraic proof of this deduction. We also do not have an explicit description of the left-hand map for $v>1$.

We now turn our attention to the right-hand vertical map in (3.1.1). To this end, we consider the cyclotomic trace map

$$
\operatorname{tr}: K_{q}(\mathcal{K}, \mathbb{Z} / p) \rightarrow \mathrm{TC}_{q}^{\cdot}(\mathcal{V} \mid \mathcal{K} ; p, \mathbb{Z} / p)
$$

from $K$-theory to topological cyclic homology; see [10, Sect. 1]. The right-hand side is related to $\mathrm{TR}_{*}^{*}(\mathcal{V} \mid \mathcal{K} ; p, \mathbb{Z} / p)$ by a natural exact sequence of pro-abelian groups

$$
0 \rightarrow \mathrm{TR}_{q+1}^{\cdot}(\mathcal{V} \mid \mathcal{K} ; p, \mathbb{Z} / p)_{F=1} \stackrel{\delta}{\rightarrow} \mathrm{TC}_{q}^{\cdot}(\mathcal{V} \mid \mathcal{K} ; p, \mathbb{Z} / p) \rightarrow \mathrm{TR}_{q}^{\cdot}(\mathcal{V} \mid \mathcal{K} ; p, \mathbb{Z} / p)^{F=1} \rightarrow 0 .
$$

We consider the composition of the left-hand map and the canonical map

$$
\left(\bar{W} \cdot \Omega_{\left(\mathcal{V}, M_{\mathcal{V}}\right)}^{q+1}\right)_{F=1} \rightarrow \mathrm{TR}_{q+1}^{\cdot}(\mathcal{V} \mid \mathcal{K} ; p, \mathbb{Z} / p)_{F=1} .
$$

Proposition 3.2.3. For all integers $q$, the cyclotomic trace and the map $\delta$ give rise to a natural isomorphism of pro-abelian groups

$$
K_{q}(\mathcal{K}, \mathbb{Z} / p) \oplus\left(\bar{W} \cdot \Omega_{\left(\mathcal{V}, M_{\mathcal{V}}\right)}^{q+1}\right)_{F=1} \stackrel{\sim}{\longrightarrow} \mathrm{TC}_{q}^{\cdot}(\mathcal{V} \mid \mathcal{K} ; p, \mathbb{Z} / p) .
$$

Proof. We consider the following diagram of pro-abelian groups, where the horizontal maps are given by the cyclotomic trace on the first summand and the boundary map on the second summand, and where the vertical maps are induced by the canonical projection:

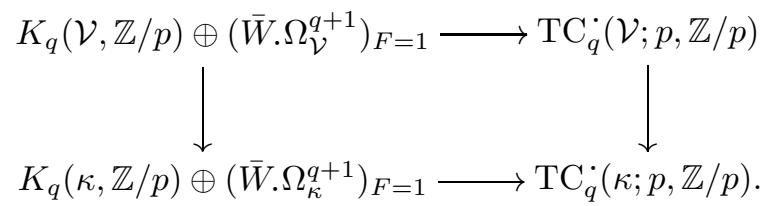

The lower horizontal map is an isomorphism by [7, Thm. 4.2.2], and we claim that also the top horizontal map is an isomorphism. Indeed, by Addendum 2.1.3. the left-hand vertical map induces an isomorphism of the second summand of the domain onto the second summand of the target, so the claim follows from the fact that the map of relative groups induced by the cyclotomic trace

$$
K_{q}(\mathcal{V}, \mathfrak{m} \mathcal{V}, \mathbb{Z} / p) \stackrel{\sim}{\longrightarrow} \mathrm{TC}_{q}^{\cdot}(\mathcal{V}, \mathfrak{m} \mathcal{V} ; p, \mathbb{Z} / p)
$$


is an isomorphism of pro-abelian groups. The latter statement, in turn, is proved in [20, 23, 22, and [5, Thm. 2.1.1].

We recall that [10, Addendum 1.5.7] gives a map of localization sequences

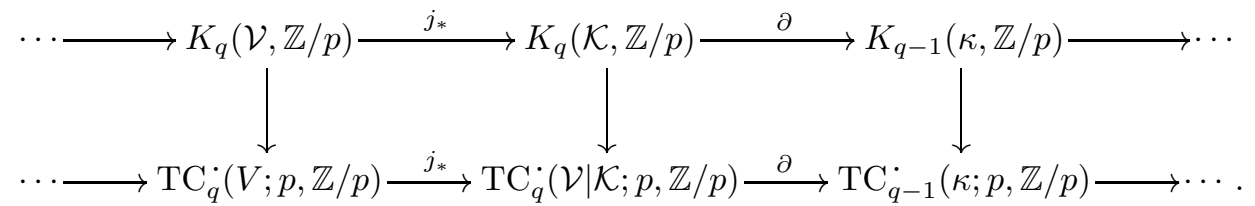

Moreover, it follows from Addendum 1.3.6 and Thm. 2.1.2(i) that the upper row in the following diagram is exact:

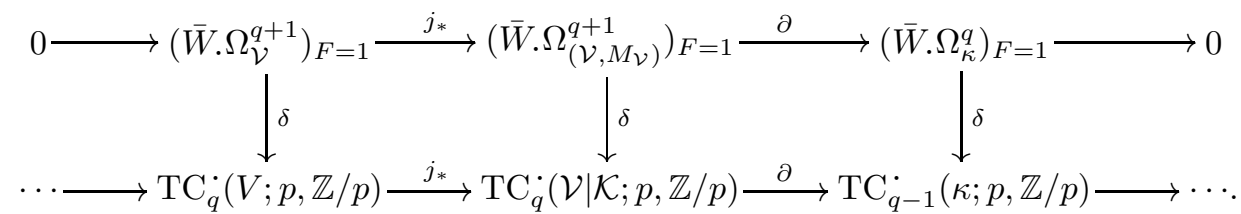

We claim that the diagram commutes. Indeed, the left-hand square commutes by the universal property of the de Rham-Witt complex, and the description of the upper horizontal map $\partial$ in terms of local coordinates shows that, in order to show that the right-hand square commutes, it suffices to show that the lower horizontal map $\partial$ takes $d \log \pi$ to 1 . But this follows from the definition of $d \log \pi$ and from the commutativity of the right-hand square in the previous diagram.

Finally, we combine the two diagrams above as follows. The two diagrams give rise to a map of long-exact sequences from the sum of the upper rows in the two diagrams to the common lower row in the two diagrams. We showed in the beginning of the proof that, in this map of long-exact sequences, two out of three maps are isomorphisms of pro-abelian groups. The third map is the map of the statement. This completes the proof.

Proof of Thm. B. We first note that if the statement is proved in the basic case $v=1$, then the general case $v \geqslant 1$ follows inductively by using that the coefficient sequence breaks up into short-exact sequences

$$
0 \rightarrow K_{q}(\mathcal{K}, \mathbb{Z} / p) \rightarrow K_{q}\left(\mathcal{K}, \mathbb{Z} / p^{v}\right) \rightarrow K_{q}\left(\mathcal{K}, \mathbb{Z} / p^{v-1}\right) \rightarrow 0 .
$$

Hence, it suffices to consider the case $v=1$. It follows from Propositions 3.2.1 and 3.2.3 that the left and right-hand vertical maps in (3.1.1) are surjections onto the domain and target, respectively, of the canonical map

$$
\left(\bar{W} \cdot \Omega_{\left(\mathcal{V}, M_{\mathcal{V}}\right)}^{*} \otimes S_{\mathbb{Z} / p}\left(\mu_{p}\right)\right)^{F=1} \stackrel{\sim}{\longrightarrow} \mathrm{TR}_{*}^{*}(\mathcal{V} \mid \mathcal{K} ; p, \mathbb{Z} / p)^{F=1} .
$$

The two propositions further identify the kernel of both of the vertical maps in (3.1.1) with the direct sum

$$
\bigoplus_{s \geqslant 1}\left(\bar{W} \cdot \Omega_{\left(\mathcal{V}, M_{\mathcal{V}}\right)}^{q+1-2 s} \otimes \mu_{p}^{\otimes s}\right)_{F=1} .
$$

It remains to show that the map between the two kernels induced by the upper horizontal map in (3.1.1) is an isomorphism. This, in turn, is equivalent to showing that the following diagram (3.2.4) of pro-abelian groups commutes. The three unmarked maps are as follows: the upper horizontal map is induced by the lower 
horizontal map in (3.1.1); the lower horizontal map is the composition of the canonical map from Milnor $K$-theory to algebraic $K$-theory followed by the cyclotomic trace; and the left-hand vertical map is the left-hand map in Prop. 3.2.1

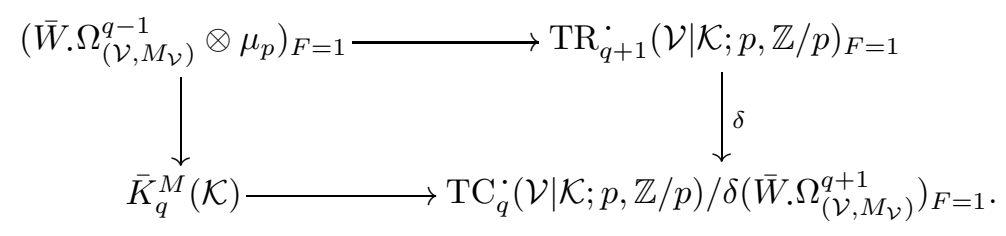

It follows from Addendum 2.1.3 that every element of the upper left-hand term can be written in the form $[a] d \log x_{1} \ldots d \log x_{q-1} \otimes \zeta$, and since all maps in (3.2.4) are $\bar{K}_{*}^{M}(\mathcal{K})$-linear, we can further assume that $q=1$. Hence, it suffices to prove the following Prop. 3.2.5.

Proposition 3.2.5. The image of $[a] \otimes \zeta$ by the composite map

$$
W .(\mathcal{V}) \otimes \mu_{p} \rightarrow \mathrm{TR}_{2}(\mathcal{V} \mid \mathcal{K} ; p, \mathbb{Z} / p) \stackrel{\delta}{\rightarrow} \mathrm{TC}_{1}(\mathcal{V} \mid \mathcal{K} ; p, \mathbb{Z} / p)
$$

is congruent, modulo $\delta\left(\bar{W} \cdot \Omega_{\left(\mathcal{V}, M_{\mathcal{V}}\right)}^{2}\right)_{F=1}$, to $d \log \left(1+a(1-\zeta)^{p}\right)$.

Proof. We may assume that the discrete valuation ring $\mathcal{V}$ is complete. Indeed, the canonical map for $\mathcal{V}$ to the completion of $\mathcal{V}$ induces an isomorphism of all three terms in the statement. The line of proof is similar to that of [10, Addendum 3.3.9]. We apply op. cit., Lemma 3.3.10, to the $3 \times 3$-diagram of cofibration sequences

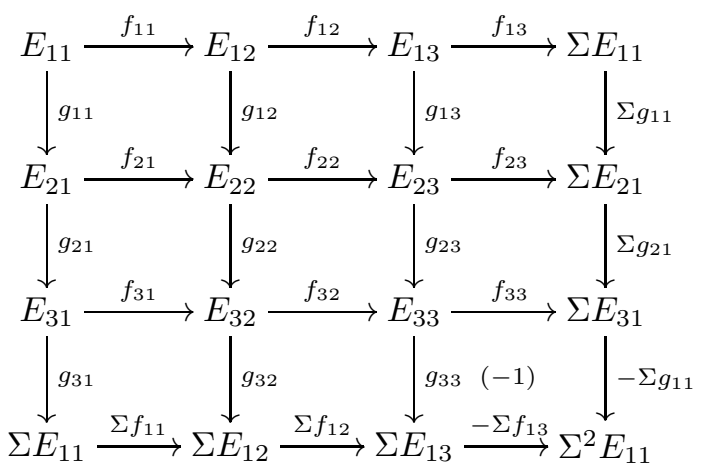

obtained as the smash product of the coefficient sequence

$$
S^{0} \stackrel{p}{\rightarrow} S^{0} \rightarrow M_{p} \stackrel{\beta}{\rightarrow} S^{1}
$$

as the first smash factor and the fundamental cofibration sequence

$$
\mathrm{TC}^{n}(\mathcal{V} \mid \mathcal{K} ; p) \rightarrow \mathrm{TR}^{n}(\mathcal{V} \mid \mathcal{K} ; p) \stackrel{R-F}{\longrightarrow} \mathrm{TR}^{n-1}(\mathcal{V} \mid \mathcal{K} ; p) \stackrel{\delta}{\rightarrow} \Sigma \mathrm{TC}^{n}(\mathcal{V} \mid \mathcal{K} ; p)
$$

as the second smash factor. We recall from op. cit., Lemma 3.3.10, that if we are given classes $e_{i j} \in \pi_{*}\left(E_{i j}\right)$ such that $g_{33}\left(e_{33}\right)=f_{12}\left(e_{12}\right)$ and $f_{33}\left(e_{33}\right)=g_{21}\left(e_{21}\right)$, then the sum $f_{21}\left(e_{21}\right)+g_{12}\left(e_{12}\right)$ is in the image of $\pi_{*}\left(E_{11}\right) \rightarrow \pi_{*}\left(E_{22}\right)$. In the case at hand, we consider the class

$$
e_{33}=[a]_{n-1}^{p} \cdot b_{\zeta} \in \pi_{2}\left(E_{33}\right)=\pi_{2}\left(M_{p} \wedge \mathrm{TR}^{n-1}(\mathcal{V} \mid \mathcal{K} ; p)\right) .
$$

We wish to show that the image $e_{31}$ of $e_{33}$ by the map

$$
f_{33 *}=(\mathrm{id} \wedge \delta)_{*}: \pi_{2}\left(M_{p} \wedge \mathrm{TR}^{n-1}(\mathcal{V} \mid \mathcal{K} ; p)\right) \rightarrow \pi_{1}\left(M_{p} \wedge \mathrm{TC}^{n}(\mathcal{V} \mid \mathcal{K} ; p)\right)
$$


is congruent, modulo the image of $\pi_{2}\left(E_{23}\right) \rightarrow \pi_{1}\left(E_{31}\right)$, to the class

$$
e_{31}^{\prime}=d \log _{n}\left(1+a(1-\zeta)^{p}\right) .
$$

We shall use repeatedly that the canonical map

$$
W_{n} \Omega_{\left(\mathcal{V}, M_{\mathcal{V}}\right)}^{q} \rightarrow \mathrm{TR}_{q}^{n}(\mathcal{V} \mid \mathcal{K} ; p)=\pi_{q}\left(S^{0} \wedge \mathrm{TR}^{n}(\mathcal{V} \mid \mathcal{K} ; p)\right)
$$

is an isomorphism, if $q \leqslant 2$. This was proved in [10, Thm. 3.3.8] for $\mathcal{V}=V$. The general case follows from this by [11, Thms. B and C].

By the definition of the Bott element, the image of $e_{33}$ by the map

$$
g_{33 *}=(\beta \wedge \mathrm{id})_{*}: \pi_{2}\left(M_{p} \wedge \mathrm{TR}^{n-1}(\mathcal{V} \mid \mathcal{K} ; p)\right) \rightarrow \pi_{1}\left(S^{0} \wedge \mathrm{TR}^{n-1}(\mathcal{V} \mid \mathcal{K} ; p)\right)
$$

is equal to the class

$$
e_{13}=[a]_{n-1}^{p} d \log _{n-1} \zeta .
$$

Since we assume that $\mathcal{V}$ is $\mathfrak{m} \mathcal{V}$-adically complete, the proof of Lemma 2.1.1 shows that this class is in the image of

$$
f_{12 *}=R-F: W_{n} \Omega_{\left(\mathcal{V}, M_{\mathcal{V}}\right)}^{1} \rightarrow W_{n-1} \Omega_{\left(\mathcal{V}, M_{\mathcal{V}}\right)}^{1} .
$$

Indeed, the class $e_{13}$ is contained in $\operatorname{Fil}_{U}^{2 e^{\prime \prime}} W_{n-1} \Omega_{\left(\mathcal{V}, M_{\mathcal{V}}\right)}^{1}$ and $e^{\prime \prime} \geqslant 1$. This also shows that the class $e_{31}$, which we wish to determine, is contained in the image of the map $g_{21 *}: \pi_{1}\left(E_{21}\right) \rightarrow \pi_{1}\left(E_{31}\right)$.

We write $\zeta=1+u \pi^{e^{\prime \prime}}$ with $u \in \mathcal{V}^{*}$ a unit and consider the class

$$
e_{12}=-\sum_{s=0}^{n-1} \sum_{t=0}^{s} d V^{s}\left([a]_{n-s}^{p^{t}}[u]_{n-s}[\pi]_{n-s}^{e^{\prime \prime}}\right) .
$$

Sublemma 3.2.6. $f_{12 *}\left(e_{12}\right) \in e_{13}+\mathrm{Fil}_{U}^{4 e^{\prime \prime}} W_{n-1} \Omega_{\left(\mathcal{V}, M_{\mathcal{V}}\right)}^{1}$.

Proof. We assume that $u=1$ (the general case is only notationally more complicated) and calculate

$$
\begin{aligned}
(R-F) e_{12}= & -\sum_{s=0}^{n-2} \sum_{t=0}^{s} d V^{s}\left([a]_{n-1-s}^{p^{t}}[\pi]_{n-1-s}^{e^{\prime \prime}}\right) \\
& +\sum_{s=1}^{n-1} \sum_{t=0}^{s} d V^{s-1}\left([a]_{n-s}^{p^{t}}[\pi]_{n-s}^{e^{\prime \prime}}\right)+F d\left([a]_{n}[\pi]_{n}^{e^{\prime \prime}}\right) \\
= & \sum_{s=0}^{n-2} d V^{s}\left([a]_{n-1-s}^{p^{s+1}}[\pi]_{n-1-s}^{e^{\prime \prime}}\right)+F d\left([a]_{n}[\pi]_{n}^{e^{\prime \prime}}\right) \\
= & \sum_{s=0}^{n-2} d\left([a]_{n-1}^{p} V^{s}\left([\pi]_{n-1-s}^{e^{\prime \prime}}\right)\right)+F d\left([a]_{n}[\pi]_{n}^{e^{\prime \prime}}\right) \\
= & \sum_{s=0}^{n-2}[a]_{n-1}^{p} d V^{s}\left([\pi]_{n-1-s}^{e^{\prime \prime}}\right) \\
& +\sum_{s=0}^{n-2} p[a]_{n-1}^{p-1} d[a]_{n-1} \cdot V^{s}\left([\pi]_{n-1-s}^{e^{\prime \prime}}\right) \\
& +[a]_{n-1}^{p-1}[\pi]_{n-1}^{e} d\left([a]_{n-1}[\pi]_{n-1}^{e^{\prime \prime}}\right) .
\end{aligned}
$$


The summands in the last two lines lie in $p \mathrm{Fil}_{U}^{2 e^{\prime \prime}}=\mathrm{Fil}_{U}^{2\left(e+e^{\prime \prime}\right)}$ and the sum in the third to last line is congruent to $[a]_{n-1}^{p} d \log _{n-1}\left(1+\pi^{e^{\prime \prime}}\right)$ modulo Fil ${ }_{U}^{4 e^{\prime \prime}}$ by Lemma 1.2.3 This completes the proof.

It follows from Lemma 2.1.1 that for $m \geqslant 2$,

$$
(R-F)^{-1}\left(\operatorname{Fil}_{U}^{m} W_{n-1} \Omega_{\left(\mathcal{V}, M_{\mathcal{V}}\right)}^{q}\right)=\operatorname{Fil}_{U}^{m} W_{n} \Omega_{\left(\mathcal{V}, M_{\mathcal{V}}\right)}^{q}+\operatorname{ker}(R-F),
$$

and hence, Sublemma 3.2 .6 implies that

$$
f_{12 *}^{-1}\left(e_{13}+\operatorname{Fil}_{U}^{4 e^{\prime \prime}} W_{n-1} \Omega_{\left(\mathcal{V}, M_{\mathcal{V}}\right)}^{1}\right)=e_{12}+\mathrm{Fil}_{U}^{4 e^{\prime \prime}} W_{n} \Omega_{\left(\mathcal{V}, M_{\mathcal{V}}\right)}^{1}+\operatorname{im}\left(f_{11 *}\right) .
$$

We next consider the image of this subset by the map

$$
g_{12 *}=p: W_{n} \Omega_{\left(\mathcal{V}, M_{\mathcal{V}}\right)}^{1} \rightarrow W_{n} \Omega_{\left(\mathcal{V}, M_{\mathcal{V}}\right)}^{1} .
$$

Sublemma 3.2.8. The subset $g_{12 *}\left(f_{12 *}^{-1}\left(e_{13}+\mathrm{Fil}_{U}^{4 e^{\prime \prime}} W_{n-1} \Omega_{\left(\mathcal{V}, M_{\mathcal{V}}\right)}^{1}\right)\right)$ is equal to the subset $d \log _{n}\left(1+a(1-\zeta)^{p}\right)+\operatorname{Fil}_{U}^{2\left(e^{\prime}+e^{\prime \prime}\right)} W_{n} \Omega_{\left(\mathcal{V}, M_{\mathcal{V}}\right)}^{1}+\operatorname{im}\left(g_{12 *} f_{11 *}\right)$.

Proof. We again assume $u=1$ and recall from Lemma 1.2 .2 that

$$
p \operatorname{Fil}_{U}^{4 e^{\prime \prime}} W_{n} \Omega_{\left(\mathcal{V}, M_{\mathcal{V}}\right)}^{1}=\operatorname{Fil}_{U}^{2\left(e^{\prime}+e^{\prime \prime}\right)} W_{n} \Omega_{\left(\mathcal{V}, M_{\mathcal{V}}\right)}^{1} .
$$

Hence, in view of the equation (3.2.7), it will suffice to prove that

$$
g_{12 *}\left(e_{12}\right) \in d \log _{n}\left(1+a(1-\zeta)^{p}\right)+\operatorname{Fil}_{U}^{2\left(e^{\prime}+e^{\prime \prime}\right)} W_{n} \Omega_{\left(\mathcal{V}, M_{\mathcal{V}}\right)}^{1} .
$$

To this end, we use that in $W_{m}(\mathcal{V})$,

$$
[\pi]_{m}^{e^{\prime}} \equiv p\left(-\sum_{v=0}^{m-1} V^{v}\left([\pi]_{m-v}^{e^{\prime \prime}}\right)\right)
$$

modulo $W_{m}\left(\mathfrak{m}^{2 e^{\prime}} \mathcal{V}\right)$. If we rewrite

$$
e_{12}=\sum_{s=0}^{n-1} d V^{s}\left([a]_{n-s}\left(-\sum_{v=0}^{n-s-1} V^{v}\left([\pi]_{n-s-v}^{e^{\prime \prime}}\right)\right)\right),
$$

this implies that $g_{12 *}\left(e_{12}\right)$ is congruent, modulo $\mathrm{Fil}_{U}^{4 e^{\prime}} W_{n} \Omega_{\left(\mathcal{V}, M_{\mathcal{V}}\right)}^{1}$, to the sum

$$
\sum_{s=0} d V^{s}\left([a]_{n-s}[\pi]_{n-s}^{e^{\prime}}\right) .
$$

Finally, Lemma 1.2 .3 shows that this sum is congruent, modulo $\operatorname{Fil}_{U}^{4 e^{\prime}} W_{n} \Omega_{\left(\mathcal{V}, M_{\mathcal{V}}\right)}^{1}$ to the class $e_{22}=d \log _{n}\left(a(1-\zeta)^{p}\right)$.

Recall that the map

$$
\bar{f}_{21 *}: \pi_{1}\left(E_{21}\right) / \operatorname{im}\left(f_{23 *}\right) \hookrightarrow \pi_{1}\left(E_{22}\right)
$$

induced by $f_{21 *}$ is identified with the canonical inclusion

$$
\left(W_{n} \Omega_{\left(\mathcal{V}, M_{\mathcal{V}}\right)}^{1}\right)^{F=1} \hookrightarrow W_{n} \Omega_{\left(\mathcal{V}, M_{\mathcal{V}}\right)}^{1} .
$$

We can now conclude that $\bar{f}_{21 *}^{-1}\left(g_{12 *}\left(f_{12 *}^{-1}\left(e_{13}\right)\right)\right)$ is contained in

$$
d \log _{n}\left(1+a(1-\zeta)^{p}\right)+\operatorname{Fil}_{U}^{2\left(e^{\prime}+e^{\prime \prime}\right)}\left(W_{n} \Omega_{\left(\mathcal{V}, M_{\mathcal{V}}\right)}^{1}\right)^{F=1}+\operatorname{im}\left(\bar{g}_{11 *}\right) .
$$

The image of this set by the map

$$
\bar{g}_{21 *}: \pi_{1}\left(E_{21}\right) / \operatorname{im}\left(f_{23 *}\right) \rightarrow \pi_{1}\left(E_{31}\right) / \operatorname{im}\left(g_{21 *} f_{23 *}\right)
$$


is equal to the class of $d \log _{n}\left(1+a(1-\zeta)^{p}\right)$, provided that

$$
\operatorname{Fil}_{U}^{2\left(e^{\prime}+e^{\prime \prime}\right)}\left(W_{n} \Omega_{\left(\mathcal{V}, M_{\mathcal{V}}\right)}^{1}\right)^{F=1} \subset p\left(W_{n} \Omega_{\left(\mathcal{V}, M_{\mathcal{V}}\right)}^{1}\right)^{F=1} .
$$

We shall prove in Cor. 3.2 .10 below that this is almost true. More precisely, we will show that given $n \geqslant 1$, there exists $m \geqslant n$ such that the left-hand side is contained in the image of the composite

$$
p\left(W_{m} \Omega_{\left(\mathcal{V}, M_{\mathcal{V}}\right)}^{1}\right)^{F=1} \hookrightarrow W_{m} \Omega_{\left(\mathcal{V}, M_{\mathcal{V}}\right)}^{1} \stackrel{R^{m-n}}{\longrightarrow} W_{n} \Omega_{\left(\mathcal{V}, M_{\mathcal{V}}\right)}^{1} .
$$

The proof of this will occupy the rest of this section. We may then conclude that given $n \geqslant 1$, there exists $m \geqslant n$ such that the map of the statement takes $[a]_{m} \otimes \zeta$ to $d \log _{n}\left(1+a(1-\zeta)^{p}\right)$. The proposition follows.

Lemma 3.2.9. The map that to $x \otimes \zeta$ assigns $x d \log \zeta$ is an isomorphism of proabelian groups

$$
W .(\mathfrak{m} \mathcal{V}) \otimes \mu_{p} \stackrel{\sim}{\longrightarrow}\left(\operatorname{Fil}_{U}^{2\left(e^{\prime \prime}+1\right)} W \cdot \Omega_{\left(\mathcal{V}, M_{\mathcal{V}}\right)}^{1}\right)[p] .
$$

Proof. It follows from [11, Thm. E] that the map

$$
W .(\mathcal{V}) \otimes \mu_{p} \rightarrow W . \Omega_{\left(\mathcal{V}, M_{\mathcal{V}}\right)}^{1}[p]
$$

that to $x \otimes \zeta$ associates $x d \log \zeta$ is an isomorphism of pro-abelian groups. This map factors as the composite

$$
W .(\mathcal{V}) \otimes \mu_{p} \rightarrow\left(\operatorname{Fil}_{U}^{2 e^{\prime \prime}} W . \Omega_{\left(\mathcal{V}, M_{\mathcal{V}}\right)}^{1}\right)[p] \hookrightarrow W \cdot \Omega_{\left(\mathcal{V}, M_{\mathcal{V}}\right)}^{1}[p],
$$

and since the right-hand map is injective, both maps are necessarily isomorphisms. We wish to conclude that the map of the statement is an isomorphism. To this end we consider the following diagram of pro-abelian groups with exact columns.

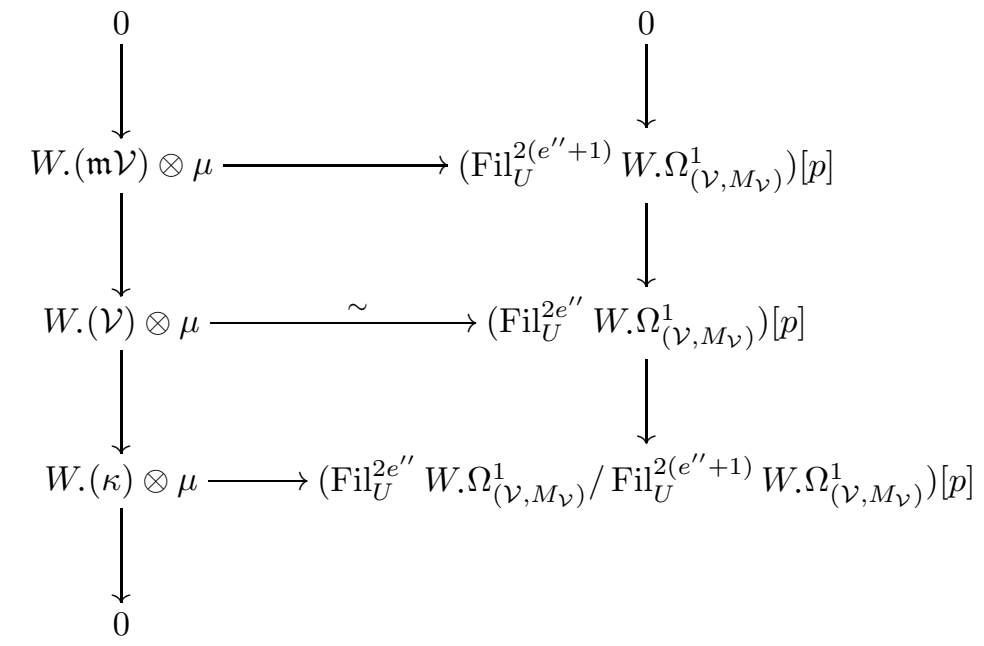

It suffices to show that the lower horizontal map is injective. To prove this, we compose with the canonical inclusion

$$
\left(\operatorname{Fil}_{U}^{2 e^{\prime \prime}} W \cdot \Omega_{\left(\mathcal{V}, M_{\mathcal{V}}\right)}^{1} / \operatorname{Fil}_{U}^{2\left(e^{\prime \prime}+1\right)} W \cdot \Omega_{\left(\mathcal{V}, M_{\mathcal{V}}\right)}^{1}\right)[p] \hookrightarrow W \cdot \Omega_{\left(\mathcal{V}, M_{\mathcal{V}}\right)}^{1} / \operatorname{Fil}_{U}^{2\left(e^{\prime \prime}+1\right)} W \cdot \Omega_{\left(\mathcal{V}, M_{\mathcal{V}}\right)}^{1}
$$

and use the isomorphism of Lemma 1.2.1

$$
W \cdot \Omega_{\left(\mathcal{V}, M_{\mathcal{V}}\right)}^{1} / \mathrm{Fil}_{U}^{2\left(e^{\prime \prime}+1\right)} W \cdot \Omega_{\left(\mathcal{V}, M_{\mathcal{V}}\right)}^{1} \stackrel{\sim}{\longrightarrow} W \cdot \Omega_{\left(\mathcal{V}_{e^{\prime \prime}+1}, M_{e^{\prime \prime}+1}\right)}^{1} .
$$


The resulting map

$$
W_{n}(\kappa) \otimes \mu_{p} \rightarrow W \cdot \Omega_{\left(\mathcal{V}_{e^{\prime \prime}+1}, M_{e^{\prime \prime}+1}\right)}^{1}
$$

takes $a \otimes \zeta$ to $\tilde{a} d \log _{n} \bar{\zeta}$, where $\tilde{a} \in W_{n}\left(\mathcal{V}_{e^{\prime \prime}+1}\right)$ is any lifting of $a \in W_{n}(\kappa)$. The ring $\mathcal{V}_{e^{\prime \prime}+1}$ is isomorphic to the truncated polynomial ring $\kappa[t] /\left(t^{e^{\prime \prime}+1}\right)$, and we can choose the isomorphism such that the induced map of residue fields is the identity map. The image of $\bar{\zeta}$ by this isomorphism has the form $1+u t^{e^{\prime \prime}}$, where $u \in \kappa^{*}$ is a unit. (Since $\zeta \in V$, we can even assume that $u \in k^{*}$.) Hence, it follows from Lemma 1.2 .3 that the composition

$$
W_{n}(\kappa) \otimes \mu_{p} \rightarrow W_{n} \Omega_{\left(\mathcal{V}_{e^{\prime \prime}+1}, M_{e^{\prime \prime}+1}\right)}^{1} \stackrel{\sim}{\longrightarrow} W_{n} \Omega_{\left(\kappa[t] /\left(t^{e^{\prime \prime}+1}\right), \mathbb{N}_{0}\right)}^{1}
$$

is equal to the $W_{n}(\kappa)$-linear map that takes $1 \otimes \zeta$ to the sum

$$
d \log _{n} \bar{\zeta}=\sum_{s=0}^{n-1} d V^{s}\left([u]_{n-s}[t]_{n-s}^{e^{\prime \prime}}\right)
$$

The domain of this map is given by

$$
W_{n}(\kappa) / p W_{n}(\kappa) \otimes \mu_{p}=W_{n}(\kappa) / V F W_{n}(\kappa) \otimes \mu_{p}=W_{n}(\kappa) / V W_{n}\left(\kappa^{p}\right) \otimes \mu_{p},
$$

and the target is given by Prop. A.1.1 below. We must show that if $n$ is sufficiently large, then for all $a \in W_{n}(\kappa)$, the product

$$
\Theta=a d \log _{n} \bar{\zeta}=\left(\sum_{r=0}^{n-1} V^{r}\left(\left[a_{r}\right]_{n-r}\right)\right) \cdot\left(\sum_{s=0}^{n-1} d V^{s}\left([u]_{n-s}[t]_{n-s}^{e^{\prime \prime}}\right)\right)
$$

is equal to zero if and only if $a_{0}=0$ and $a_{r} \in \kappa^{p}$, for all $1 \leqslant r<n$. We write $e^{\prime \prime}=p^{v} i$ with $i$ prime to $p$ and proceed to rewrite the summands of $\Theta=\sum \Theta_{r, s}$ in the form of Prop. A.1.1. We first note that since $d$ is a derivation,

$$
\Theta_{r, s}=d\left(V^{r}\left(\left[a_{r}\right]_{n-r}\right) \cdot V^{s}\left([u]_{n-s}[t]_{n-s}^{p^{v} i}\right)\right)-d V^{r}\left(\left[a_{r}\right]_{n-r}\right) \cdot V^{s}\left([u]_{n-s}[t]_{n-s}^{p^{v} i}\right),
$$

for all $0 \leqslant r, s<n$.

Suppose first that $r>0$. If $0 \leqslant s \leqslant v$ and $s \geqslant r$, we get

$$
\begin{aligned}
\Theta_{r, s}= & p^{r} d V^{s}\left(\left[a_{r}\right]_{n-s}^{p^{s-r}}[u]_{n-s}\right) \cdot[t]_{n}^{p^{v-s} i} \\
& +p^{r+v-s} i V^{s}\left(\left[a_{r}\right]_{n-s}^{p^{s-r}}[u]_{n-s}\right) \cdot[t]_{n}^{p^{v-s}} d \log _{n} t \\
& -V^{s}\left(\left[a_{r}\right]_{n-s}^{p^{s-r}} d \log _{n-s} a_{r} \cdot[u]_{n-s}\right) \cdot[t]_{n}^{p^{v-s} i}
\end{aligned}
$$

The first summand on the right-hand side is zero, since

$$
p^{r} d V^{s}\left(\left[a_{r}\right]_{n-s}^{p^{s-r}}[u]_{n-s}\right) \in \mathrm{Fil}^{s+r} W_{n} \Omega_{\kappa}^{1}
$$

and $p^{r+s} \cdot p^{s-v} i \geqslant e^{\prime \prime}+1$, and the second term is zero for similar reasons. The third term is zero if and only if

$$
V^{s}\left(\left[a_{r}\right]_{n-s}^{p^{s-r}} d \log _{n-s} a_{r} \cdot[u]_{n-s}\right) \in \mathrm{Fil}^{s+1} W_{n} \Omega_{\kappa}^{1},
$$

and this happens if and only if $a_{r} \in \kappa^{p}$. Indeed, the filtration of the groups $W_{n} \Omega_{\kappa}^{q}$ is known completely by [13, Prop. I.2.12]. If $0 \leqslant s \leqslant v$ and $s<r$, we have

$$
\begin{aligned}
\Theta_{r, s}= & p^{s} d V^{s}\left(V^{r-s}\left(\left[a_{r}\right]_{n-r}\right)[u]_{n-s}\right) \cdot[t]_{n}^{p^{v-s} i} \\
= & p^{v} i V^{s}\left(V^{r-s}\left(\left[a_{r}\right]_{n-r}\right)[u]_{n-s}\right) \cdot[t]_{n}^{p^{v-s} i} d \log _{n} t \\
& -V^{s}\left(d V^{r-s}\left(\left[a_{r}\right]_{n-r}\right)[u]_{n-s}\right) \cdot[t]_{n}^{p^{v-s}},
\end{aligned}
$$


and all three terms are zero, since $p^{r} \cdot p^{v-s} i \geqslant e^{\prime \prime}+1$. If $0 \leqslant v<s$ and $s \geqslant r$,

$$
\begin{aligned}
\Theta_{r, s}= & p^{r} d V^{s-v}\left(V^{v}\left(\left[a_{r}\right]_{n-s}^{p^{s-r}}[u]_{n-s}\right) \cdot[t]_{n-s+v}^{i}\right) \\
& -V^{s-v}\left(V^{v}\left(\left[a_{r}\right]_{n-s}^{p^{s-r}} d \log _{n-s} a_{r} \cdot[u]_{n-s}\right) \cdot[t]_{n-s+v}^{i}\right) .
\end{aligned}
$$

The first term is zero, since $p^{v+r} i \geqslant e^{\prime \prime}+1$, and the second term is equal to zero if and only if $a_{r} \in \kappa^{p}$. Finally, if $0 \leqslant v<s$ and $s<r$, we have

$$
\begin{aligned}
\Theta_{r, s}= & p^{s} d V^{s-v}\left(V^{v}\left(V^{r-s}\left(\left[a_{r}\right]_{n-r}\right)[u]_{n-s}\right) \cdot[t]_{n-s+v}^{i}\right) \\
& -V^{s-v}\left(V^{v}\left(d V^{r-s}\left(\left[a_{r}\right]_{n-r}\right)[u]_{n-s}\right) \cdot[t]_{n-s+v}^{i}\right)
\end{aligned}
$$

and both terms are zero since $p^{r-s+v} i \geqslant e^{\prime \prime}+1$.

We next evaluate the remaining summands $\Theta_{0, s}$. If $0 \leqslant s \leqslant v$, we have

$$
\begin{aligned}
\Theta_{0, s}= & d V^{s}\left(\left[a_{0}\right]_{n-s}^{p^{s}}[u]_{n-s}\right) \cdot[t]_{n}^{p^{v-s} i} \\
& +p^{v-s} i V^{s}\left(\left[a_{0}\right]_{n-s}^{p^{s}}[u]_{n-s}\right) \cdot[t]_{n}^{p^{v-s}} d \log _{n} t \\
& -V^{s}\left(\left[a_{0}\right]_{n-s}^{p^{s}} d \log _{n-s} a_{0} \cdot[u]_{n-s}\right) \cdot[t]_{n}^{p^{v-s} i} .
\end{aligned}
$$

The first term is zero if and only if $s=0$ and $a_{0} \in \kappa^{p}$, the second term is zero if and only if $s<v$, and the last term is zero if and only if $a_{0} \in \kappa^{p}$. Finally, if $s>v$,

$$
\begin{aligned}
\Theta_{0, s}= & d V^{s-v}\left(V^{v}\left(\left[a_{0}\right]_{n-s}^{p^{s}}[u]_{n-s}\right) \cdot[t]_{n-s+v}^{i}\right) \\
& -V^{s-v}\left(V^{v}\left(\left[a_{0}\right]_{n-s}^{p^{s}} d \log _{n-s} \cdot[u]_{n-s}\right) \cdot[t]_{n-s+v}^{i}\right) .
\end{aligned}
$$

The first term is zero if and only if $a_{0}$ is zero, and the second term is zero if and only if $a_{0} \in \kappa^{p}$.

We can now show that for $n>v$, the product

$$
\Theta=\operatorname{ad} \log _{n} \bar{\zeta} \in W_{n} \Omega_{\left(\kappa[t] /\left(t^{e^{\prime \prime}+1}\right), \mathbb{N}_{0}\right)}^{1}
$$

is equal to zero if and only if $a_{0}=0$ and $a_{r} \in \kappa^{p}$, for all $1 \leqslant r<n$, as desired. To this end we use the direct sum decomposition of the de Rham-Witt group on the left-hand side exhibited by Prop. A.1.1 below. Suppose first that $1 \leqslant r<n$. Then $\Theta_{r, s}=0$ if and only if $r>s$ or $r \leqslant s$ and $a_{r} \in \kappa^{p}$. Suppose that $r \leqslant s$. Then the element $\Theta_{r, s}$ belongs to the direct summand $V^{s}\left(W_{n-s} \Omega_{\kappa}^{1}\right) \cdot[t]_{n}^{p^{v-s} i}$, if $s \leqslant v$, and to the direct summand $V^{s-v}\left(W_{n-s+v} \Omega_{\kappa}^{q} \cdot[t]_{n-s+v}^{i}\right)$, if $s>v$. In particular, two non-zero elements $\Theta_{r, s}$ and $\Theta_{r^{\prime}, s^{\prime}}$ belong to the same summand if and only if $s=s^{\prime}$. It follows that the sum

$$
\sum_{r=1}^{n-1} \sum_{s=0}^{n-1} \Theta_{r, s}=\sum_{r=1}^{n-1} \sum_{s=r}^{n-1} \Theta_{r, s}
$$

is equal to zero if and only if $a_{r} \in \kappa^{p}$, for all $1 \leqslant r<n$. A similar argument shows that no cancellation can occur between the elements $\Theta_{r, s}, 1 \leqslant r \leqslant s<n$, and the elements $\Theta_{0, s^{\prime}}, 0 \leqslant s^{\prime}<n$. Finally, $\Theta_{0, s}$ is non-zero if $a_{0}$ is non-zero and $s \geqslant v$. This completes the proof.

Corollary 3.2.10. The map induced from multiplication by $p$,

$$
p: \operatorname{Fil}_{U}^{2\left(e^{\prime \prime}+1\right)}\left(W . \Omega_{\left(\mathcal{V}, M_{\mathcal{V}}\right)}^{1}\right)^{F=1} \rightarrow \operatorname{Fil}_{U}^{2\left(e^{\prime}+1\right)}\left(W . \Omega_{\left(\mathcal{V}, M_{\mathcal{V}}\right)}^{1}\right)^{F=1}
$$

is an isomorphism of pro-abelian groups. 
Proof. We abbreviate $E^{q}=W \cdot \Omega_{\left(\mathcal{V}, M_{\mathcal{V}}\right)}^{q}$ and consider the following diagram:

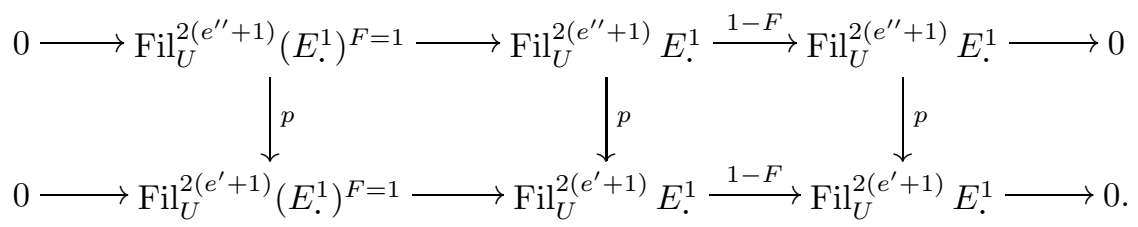

It follows from Lemma 1.2.2 that the middle and right-hand vertical maps, which are induced by multiplication by $p$, are well-defined and surjective. The left-hand vertical map is the map of kernels induced by $1-F$. This is the map of the statement. The horizontal maps $1-F$ induce a map between the kernels of the middle and right-hand vertical maps

$$
1-F: \operatorname{Fil}_{U}^{2\left(e^{\prime \prime}+1\right)} E^{1}[p] \rightarrow \operatorname{Fil}_{U}^{2\left(e^{\prime \prime}+1\right)} E^{1}[p],
$$

and Lemma 3.2 .9 shows that this map is an isomorphism of pro-abelian groups. Indeed, the map $1-F: \bar{W} .(\mathfrak{m} \mathcal{V}) \rightarrow \bar{W} .(\mathfrak{m} \mathcal{V})$ is an isomorphism, since the geometric series $1+F+F^{2}+\ldots$ converges. The corollary follows.

\section{Appendix A. Truncated polynomial algebras}

A.1. In this appendix, we give an explicit formula for the de Rham-Witt complex of a truncated polynomial algebra in terms of the de Rham-Witt complex of the coefficient ring. The formula is derived from the corresponding formula, proved in [11, Thm. B], for the de Rham-Witt complex of a polynomial algebra, and it generalizes the formula of the thesis of Kåre Nielsen [21, where the case with coefficient ring $\mathbb{F}_{p}$ was considered.

Let $A$ be a $\mathbb{Z}_{(p)}$-algebra with $p \neq 2$, and let $A[t]$ be the polynomial algebra in one variable with the pre-log structure $\alpha: \mathbb{N}_{0} \rightarrow A, \alpha(i)=t^{i}$. One can show as in [11, Thm. B] that every element $\omega^{(n)} \in W_{n} \Omega_{\left(A[t], \mathbb{N}_{0}\right)}^{q}$ can be written uniquely as

$$
\begin{aligned}
\omega^{(n)}= & \sum_{i \in \mathbb{N}_{0}}\left(a_{0, i}^{(n)}[t]_{n}^{i}+b_{0, i}^{(n)}[t]_{n}^{i} d \log _{n} t\right) \\
& +\sum_{s=1}^{n-1} \sum_{i \in I_{p}}\left(V^{s}\left(a_{s, i}^{(n-s)}[t]_{n-s}^{i}\right)+d V^{s}\left(b_{s, i}^{(n-s)}[t]_{n-s}^{i}\right)\right),
\end{aligned}
$$

where $a_{s, i}^{(m)} \in W_{m} \Omega_{A}^{q}$ and $b_{s, i}^{(m)} \in W_{m} \Omega_{A}^{q-1}$, and where $I_{p}$ denotes the set of positive integers prime to $p$. The formulas for the product, differential, and Frobenius and Verschiebung operators may be found in op. cit., Sect. 4.2. We now fix an integer $N \geqslant 1$ and consider the subgroup

$$
I_{n}^{q} \subset W_{n} \Omega_{\left(A[t], \mathbb{N}_{0}\right)}^{q}
$$

of those elements $\omega^{(n)}$ such that $a_{s, i}^{(m)} \in \mathrm{Fil}^{v} W_{m} \Omega_{A}^{q}$ and $b_{s, i}^{(m)} \in \mathrm{Fil}^{v} W_{m} \Omega_{A}^{q-1}$, for some $0 \leqslant v<m$ with $p^{v} i \geqslant N$. We consider the ring $A[t] /\left(t^{N}\right)$ with the induced pre-log structure. The following result expresses $W_{n} \Omega_{\left(A[t] /\left(t^{N}\right), \mathbb{N}_{0}\right)}^{q}$ as a direct sum of groups $W_{m-v} \Omega_{A}^{q}$ and $W_{m-v} \Omega_{A}^{q-1}$.

Proposition A.1.1. The canonical projection induces an isomorphism

$$
W_{n} \Omega_{\left(A[t], \mathbb{N}_{0}\right)}^{q} / I_{n}^{q} \stackrel{\sim}{\longrightarrow} W_{n} \Omega_{\left(A[t] /\left(t^{N}\right), \mathbb{N}_{0}\right)}^{q} .
$$


Proof. We see as in the proof of Lemma 1.2.1 that it suffices to show that $I_{n}^{*}$ is a differential graded ideal with $W_{n}\left(\left(t^{N}\right)\right) \subset I_{n}^{0}$ and that if $J_{n}^{*}$ is another differential graded ideal with $W_{n}\left(\left(t^{N}\right)\right) \subset J_{n}^{0}$, then $I_{n}^{*} \subset J_{n}^{*}$. We leave the former statement to the reader and prove the latter. We first show that elements of the form $V^{s}\left(a_{s, i}^{(n-s)}[t]_{n-s}^{i}\right)$, where $a \in \mathrm{Fil}^{v} W_{n-s} \Omega_{A}^{q}$, for some $0 \leqslant v<n-s$ with $p^{v} i \geqslant N$, are contained in $J_{n}^{q}$. By definition of the standard filtration,

$$
a_{s, i}^{(n-s)}=V^{v}(\omega)+d V^{v}\left(\omega^{\prime}\right)
$$

for some $\omega \in W_{n-s-v} \Omega_{A}^{q}$ and $\omega^{\prime} \in W_{n-s-v} \Omega_{A}^{q-1}$, and hence $V^{s}\left(a_{s, i}^{(n-s)}[t]_{n-s}^{i}\right)$ is equal to the sum

$$
V^{s+v}\left(\omega[t]_{n-s-v}^{p^{v} i}\right)+p^{s} d V^{s+v}\left(\omega^{\prime}[t]_{n-s-v}^{p^{v} i}\right)-i V^{s+v}\left(\omega^{\prime}[t]_{n-s-v}^{p^{v} i} d \log t\right) .
$$

We consider the left-hand term. By [11, Thm. A], the canonical map

$$
\Omega_{W_{n}(A)}^{q} \rightarrow W_{n} \Omega_{A}^{q}
$$

is surjective. This shows that $\omega$ can be written as a sum of elements of the form $x_{0} d x_{1} \ldots d x_{q}$, where $x_{0}, \ldots, x_{q} \in W_{n-s-v}(A)$. But

$$
V^{s+v}\left(x_{0} d x_{1} \ldots d x_{q}[t]_{n-s-v}^{p^{v} i}\right)=V^{s+v}\left(x_{0}[t]_{n-s-v}^{p^{i} v}\right) d V^{s+v}\left(x_{1}\right) \ldots d V^{s+v}\left(x_{q}\right),
$$

which is contained in $J_{n}^{q}$, and hence $V^{s+v}\left(\omega[t]_{n-s-v}^{p^{i} v}\right)$, too, is contained in $J_{n}^{q}$. One shows in a similar manner that $d V^{s+v}\left(\omega^{\prime}[t]_{n-s-v}^{p^{v}}\right)$ and $V^{s+v}\left(\omega^{\prime}[t]_{n-s-v}^{p^{v} i} d \log t\right)$ are contained in $J_{n}^{q}$. Hence $V^{s}\left(a_{s, i}^{(n-s)}[t]_{n-s}^{i}\right)$ is contained in $J_{n}^{q}$. The remaining cases are treated in a completely analogous manner.

\section{Appendix B. The Steinberg Relation (By Viorel Costeanu)}

B.1. This appendix is devoted to the proof of the following general version of the Steinberg relation in the $p$-typical de Rham-Witt complex. We assume as above that the prime $p$ is odd.

Proposition B.1.1. Let $(A, M)$ be a $\log -\mathbb{Z}_{(p)}$-algebra, and let $x$ and $y$ be two elements of $M$ with the property that the sum $\alpha(x)+\alpha(y)$ in $A$ is equal to 1 . Then the product $d \log _{n} x \cdot d \log _{n} y$ in $W_{n} \Omega_{\left(A, M_{A}\right)}^{2}$ is equal to zero.

Proof. The proof is by induction on $n \geqslant 1$. The proof of the case $n=1$ and of the induction step are similar. We assume that the statement holds for $n-1$ and prove it for $n$. We have

$$
\begin{aligned}
& d \log _{n} x \cdot d \log _{n} y=\left([\alpha(x)]_{n}+[\alpha(y)]_{n}-1-[\alpha(x)]_{n}-[\alpha(y)]_{n}\right) \cdot d \log _{n} x \cdot d \log _{n} y \\
& =d[\alpha(x)]_{n} \cdot d \log _{n} y+d \log _{n} x \cdot d[\alpha(y)]_{n}+\left(1-[\alpha(x)]_{n}-[\alpha(y)]_{n}\right) d \log _{n} x \cdot d \log _{n} y .
\end{aligned}
$$

The last summand on the right-hand side is zero by induction. Indeed,

$$
1-[\alpha(x)]_{n}-[\alpha(y)]_{n}=[\alpha(x)+\alpha(y)]_{n}-[\alpha(x)]_{n}-[\alpha(y)]_{n}=V(\tau),
$$

for a unique element $\tau \in W_{n-1}(A)$, and hence

$$
\left(1-[\alpha(x)]_{n}-[\alpha(y)]_{n}\right) \cdot d \log _{n} x \cdot d \log _{n} y=V\left(\tau \cdot d \log _{n-1} x \cdot d \log _{n-1} y\right)=0 .
$$

It remains to show that $d[\alpha(x)]_{n} \cdot d \log _{n} y$ and $d \log _{n} x \cdot d[\alpha(y)]_{n}$ are zero. The proof is the same in the two cases. Lemma B.1.2 below shows that the polynomial

$$
f_{s}(X)=p^{-s}\left((1-X)^{p^{s}}-\left(1-X^{p}\right)^{p^{s-1}}\right)
$$


has integral coefficients. Moreover, one readily verifies the formula

$$
[1-a]_{n}=[1]_{n}-[a]_{n}+\sum_{0<s<n} V^{s}\left(f_{s}\left([a]_{n-s}\right)\right)
$$

by evaluating the ghost coordinates of the two sides. It follows that

$$
\begin{aligned}
& d[\alpha(y)]_{n} \cdot d \log _{n} x=d[1-\alpha(x)]_{n} \cdot d \log _{n} x \\
& =d d \log _{n} x-d d[\alpha(x)]_{n}+\sum_{0<s<n} d V^{s}\left(f_{s}\left([\alpha(x)]_{n-s}\right)\right) \cdot d \log _{n} x .
\end{aligned}
$$

The left-hand and middle term in the bottom line are zero. This completes the proof, for $n=1$. It remains to prove that the right-hand term is zero, for $n>1$. Let $c_{s, i}$ denote the coefficient of $X^{i}$ in the polynomial $f_{s}(X)$. Suppose first that $p$ does not divide $i$. Since $A$ is assumed to be a $\mathbb{Z}_{(p)}$-algebra, the integer $i$ is invertible in $W_{n}(A)$. Hence, we can write

$$
\begin{aligned}
& c_{s, i} \cdot d V^{s}\left([\alpha(x)]_{n-s}^{i}\right) \cdot d \log _{n} x=c_{s, i} \cdot d V^{s}\left([\alpha(x)]_{n-s}^{i} \cdot d \log _{n-s} x\right) \\
& =i^{-1} c_{s, i} \cdot d V^{s} d[\alpha(x)]_{n-s}=p^{s} i^{-1} c_{s, i} \cdot d d V^{s}\left([\alpha(x)]_{n-s}\right),
\end{aligned}
$$

which is zero as desired. Finally, if $p$ divides $i$, the coefficient $c_{s, i}$ is divisible by $p^{s}$ by Lemma B.1.2. Hence, we can write

$$
\begin{aligned}
& c_{s, i} \cdot d V^{s}\left([\alpha(x)]_{n-s}^{i}\right) \cdot d \log _{n} x=p^{-s} c_{s, i} \cdot V^{s} d\left([\alpha(x)]_{n-s}^{i}\right) \cdot d \log _{n} x \\
& =p^{-s} i c_{s, i} \cdot V^{s}\left([\alpha(x)]_{n-s}^{i} d \log _{n-s} x\right) \cdot d \log _{n} x \\
& =p^{-s} i c_{s, i} \cdot V^{s}\left([\alpha(x)]_{n-s}^{i}\right) \cdot d \log _{n} x \cdot d \log _{n} x,
\end{aligned}
$$

which is zero. This completes the proof.

Lemma B.1.2. The coefficient of $X^{i}$ in the polynomial

$$
(1-X)^{p^{s}}-\left(1-X^{p}\right)^{p^{s-1}}
$$

is divisible by $p^{s}$, if $p$ does not divide $i$, and by $p^{2 s}$, if $p$ divides $i$.

Proof. In general, if two elements $a$ and $b$ of a ring $R$ are congruent modulo $p R$, then their $p^{s-1}$ th powers $a^{p^{s-1}}$ and $b^{p^{s-1}}$ are congruent modulo $p^{s} R$. It follows that $(1-X)^{p^{s}}$ and $\left(1-X^{p}\right)^{p^{s-1}}$ are congruent modulo $p^{s} \mathbb{Z}[X]$. Hence, the coefficients of the polynomial $(1-X)^{p^{s}}-\left(1-X^{p}\right)^{p^{s-1}}$ are divisible by $p^{s}$. It remains to show that if $i$ is divisible by $p$, the coefficient of $X^{i}$ in this polynomial is divisible by $p^{2 s}$. If we write $i=p j$, then the coefficient in question is equal to

$$
\left(\begin{array}{c}
p^{s} \\
p j
\end{array}\right)-\left(\begin{array}{c}
p^{s-1} \\
j
\end{array}\right)=\left(\begin{array}{c}
p^{s-1} \\
j
\end{array}\right)\left(\left(\prod p^{s}-k\right) /\left(\prod k\right)-1\right),
$$

where, on the right-hand side, the products range over integers $0<k<p j$ that are not divisible by $p$. The $p$-adic valuation of the first factor on the right-hand side is at least $s-1-v_{p}(j)$. Indeed, in general, the $p$-adic valuation of the binomial coefficient $\left(\begin{array}{c}m+n \\ m\end{array}\right)$ is equal to the number of carriers in the addition of the integers $m$ and $n$ in base $p$ [17, p. 116]. We must show that the $p$-adic valuation of the second factor on the right-hand side is at least $s+v_{p}(j)+1$. Consider the polynomial

$$
f(T)=\prod(T-k),
$$

where the product ranges over integers $0<k<p j$ that are not divisible by $p$. We wish to show that the $p$-adic valuation of $\left(f\left(p^{s}\right) / f(0)\right)-1$ is at least $s+v_{p}(j)+1$, 
and since $f(0)$ is not divisible by $p$, this is equivalent to showing that the $p$-adic valuation of $f\left(p^{s}\right)-f(0)$ is at least $s+v_{p}(j)+1$. The polynomial $f(T)-f(0)$ is divisible by $T$. It suffices to show that the $p$-adic valuation of the coefficient of $T$ in this polynomial is at least $v_{p}(j)+1$. This coefficient if equal to $f^{\prime}(0)$. Since

$$
d \log f(T)=\sum \frac{d T}{T-k}
$$

we find that

$$
f^{\prime}(0)=-f(0) \cdot \sum \frac{1}{k}
$$

where the sums range over integers $0<k<p j$ that are not divisible by $p$. The number of such integers is equal to $(p-1) j$, and since $p$ is odd, this is an even number. The partial sum of the $k$ th and $(p j-k)$ th summands is equal to

$$
\frac{1}{k}+\frac{1}{p j-k}=\frac{p j}{k(p j-k)}
$$

which has $p$-adic valuation $v_{p}(j)+1$. Finally, the $p$-adic valuation of a sum of integers is at least the minimum of the $p$-adic valuations of the summands. Hence the $p$-adic valuation of $f^{\prime}(0)$ is at least $v_{p}(j)+1$ as desired.

\section{REFERENCES}

[1] A. A. Beilinson, Letter to C. Soulé, January 11, 1982.

[2] - Height pairing between algebraic cycles, $K$-theory, arithmetic and geometry (Moscow, 1984-1986), Lecture Notes in Math., vol. 1289, Springer-Verlag, 1987, pp. 1-25. MR0923131 (89h:11027)

[3] S. Bloch and K. Kato, p-adic étale cohomology, Inst. Hautes Études Sci. Publ. Math. 63 (1986), 107-152. MR0849653 (87k:14018)

[4] M. Bökstedt, W.-C. Hsiang, and I. Madsen, The cyclotomic trace and algebraic K-theory of spaces, Invent. Math. 111 (1993), 465-540. MR1202133 (94g:55011)

[5] T. Geisser and L. Hesselholt, On the K-theory and topological cyclic homology of smooth schemes over a discrete valuation ring, Trans. Amer. Math. Soc. 358 (2006), 131-145.

[6] _ On the $K$-theory of complete regular local $\mathbb{F}_{p}$-algebras, Topology (to appear).

[7] Topological cyclic homology of schemes, K-theory (Seattle, 1997), Proc. Symp. Pure Math., vol. 67, 1999, pp. 41-87. MR.1743237 (2001g:19003)

[8] L. Hesselholt, The absolute and relative de Rham-Witt complexes, Compositio Math. 141 (2005), 1109-1127.

[9] L. Hesselholt, Algebraic K-theory and trace invariants, Proceedings of the International Congress of Mathematicians, Vol. II (Beijing, 2002), Higher Ed. Press, Beijing, 2002, pp. 415-425. MR1957052 (2004d:19005)

[10] L. Hesselholt and I. Madsen, On the K-theory of local fields, Ann. of Math. 158 (2003), 1-113. MR 1998478 (2004k:19003)

[11] _ On the de Rham-Witt complex in mixed characteristic, Ann. Sci. École Norm. Sup. 37 (2004), no. 1, 1-43. MR2050204 (2005f:19005)

[12] O. Hyodo and K. Kato, Semi-stable reduction and crystalline cohomology with logarithmic poles, Périodes p-adiques, Asterisque, vol. 223, 1994, pp. 221-268. MR1293974 (95k:14034)

[13] L. Illusie, Complexe de de Rham-Witt et cohomologie cristalline, Ann. Scient. Éc. Norm. Sup. (4) 12 (1979), 501-661. MR0565469 (82d:14013)

[14] B. Kahn, Deux théorèmes de comparaison en cohomologie étale: applications, Duke Math. J. 69 (1993), 137-165. MR1201695 (94g:14009)

[15] K. Kato, Galois cohomology of complete discrete valuation fields, Algebraic $K$-theory, Part II (Oberwolfach, 1980), Lecture Notes in Math., vol. 967, Springer, Berlin-New York, 1982, pp. 215-238. MR.0689394 (84k:12006)

[16] Logarithmic structures of Fontaine-Illusie, Algebraic analysis, geometry, and number theory, Proceedings of the JAMI Inaugural Conference (Baltimore, 1988), Johns Hopkins Univ. Press, Baltimore, MD, 1989, pp. 191-224. MR1463703 (99b:14020) 
[17] E. E. Kummer, Über die Ergänzungssätze zu den allgemeinen Reciprocitätsgesetzen, J. reine angew. Math. 44 (1852), 93-146.

[18] A. Langer and T. Zink, De Rham-Witt cohomology for a proper and smooth morphism, J. Inst. Math. Jussieu 3 (2004), 231-314. MR2055710 (2005d:14027)

[19] S. Lichtenbaum, Values of zeta-functions at non-negative integers, Number theory, Lecture Notes in Math., vol. 1068, Springer-Verlag, 1983, pp. 127-138. MR0756089

[20] R. McCarthy, Relative algebraic K-theory and topological cyclic homology, Acta Math. 179 (1997), 197-222. MR.1607555 (99e:19006)

[21] K. Nielsen, On trace homology and algebraic K-theory of truncated polynomial algebras, Thesis, Aarhus University, 2001.

[22] I. A. Panin, On a theorem of Hurewicz and K-theory of complete discrete valuation rings, Math. USSR Izvestiya 29 (1987), 119-131. MR0864175 (88a:18021)

[23] A. A. Suslin, On the K-theory of local fields, J. Pure Appl. Alg. 34 (1984), 304-318. MR0772065 (86d:18010)

[24] T. Tsuji, p-adic étale cohomology and crystalline cohomology in the semi-stable reduction case, Invent. Math. 137 (1999), 233-411. MR.1705837 (2000m:14024)

Department of Mathematics, University of Southern California, los Angeles, CalIFORNIA 90089

E-mail address: geisser@math.usc.edu

Department of Mathematics, Massachusetts institute of Technology, Cambridge, Massachusetts 02139 and Department of Mathematics, Nagoya University, Nagoya, JAPAN

E-mail address: larsh@math.mit.edu; larsh@math.nagoya-u.ac.jp 